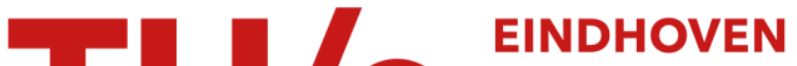 UNIVERSITY OF TECHNOLOGY
}

\section{A process-oriented methodology for evaluating the impact of IT : a proposal and an application in healthcare}

\section{Citation for published version (APA):}

Mans, R. S., Reijers, H. A., Wismeijer, D., \& Genuchten, van, M. J. I. M. (2013). A process-oriented methodology for evaluating the impact of IT : a proposal and an application in healthcare. (BPM Center Report; Vol. 1308). BPMcenter. org.

\section{Document status and date:}

Published: 01/01/2013

\section{Document Version:}

Publisher's PDF, also known as Version of Record (includes final page, issue and volume numbers)

\section{Please check the document version of this publication:}

- A submitted manuscript is the version of the article upon submission and before peer-review. There can be important differences between the submitted version and the official published version of record. People interested in the research are advised to contact the author for the final version of the publication, or visit the $\mathrm{DOI}$ to the publisher's website.

- The final author version and the galley proof are versions of the publication after peer review.

- The final published version features the final layout of the paper including the volume, issue and page numbers.

Link to publication

\section{General rights}

Copyright and moral rights for the publications made accessible in the public portal are retained by the authors and/or other copyright owners and it is a condition of accessing publications that users recognise and abide by the legal requirements associated with these rights.

- Users may download and print one copy of any publication from the public portal for the purpose of private study or research.

- You may not further distribute the material or use it for any profit-making activity or commercial gain

- You may freely distribute the URL identifying the publication in the public portal.

If the publication is distributed under the terms of Article 25fa of the Dutch Copyright Act, indicated by the "Taverne" license above, please follow below link for the End User Agreement:

www.tue.nl/taverne

Take down policy

If you believe that this document breaches copyright please contact us at:

openaccess@tue.nl

providing details and we will investigate your claim. 


\title{
A Process-oriented Methodology for Evaluating the Impact of IT: a Proposal and an Application in Healthcare
}

\author{
Ronny Mans, Eindhoven University of Technology, The Netherlands \\ Hajo Reijers, Eindhoven University of Technology, The Netherlands \\ Daniel Wismeijer, Academic Centre for Dentistry Amsterdam, The Netherlands \\ Michiel van Genuchten, Open Digital Dentistry, Switzerland
}

\begin{abstract}
In order to improve business process often Information Technologies (IT) are introduced. However, business processes are known to be complex and distributed among multiple business entities. As a result, the impact of new IT on an entire business process is typically unknown as quantitative methods for evaluation are missing. Therefore, in this paper, we propose a process-oriented methodology for evaluating the impact of IT on a business process. In our method, process mining and discrete event simulation are key ingredients. Based on automatically stored data, process mining allows for obtaining detailed knowledge on a business process, e.g., it can be discovered how a business process is actually executed. Using discrete event simulation, a model can be build which accurately mimics the discovered process and which can subsequently be used for exploring and evaluating various redesign of the same process.

Our method is evaluated by means of a detailed case study. For two complex dental processes, it turns out that the introduction of new digital technologies is largely beneficial for patients and dental lab owners, whereas for dentists there is hardly any benefit.

Keywords and phrases: Business Process Simulation, Discrete Event Simulation, Process Mining, Digital Dentistry.

\section{Introduction}

Regularly, new digital technologies emerge that afford to improve the productivity of people and organizations. Also, Information Technology (IT) usage has been demonstrated to be a key driver of organizational performance (1). Not surprisingly, the presence of computer and
\end{abstract}


information technologies in today's organizations has improved dramatically (2). With regard to assessing the usefulness or success of IT within organizations, many methodologies and frameworks exist for evaluating new ITs in terms of aspects such as usage, satisfaction, technology, performance, efficiency, costs, and so on. For example, Seddon et al proposes a two-dimensional matrix for classifying IS effectiveness measures (3), Grover et al. propose an organization level and individual level class in order to classify multiple measures (4), and the IS success model focuses on six dimensions for measuring the success of IS (5).

The introduction of IT requires a seamless integration with already existing business processes within an organization so that the operational performance of these processes is not negatively impacted. However, business processes are typically complex and distributed. That is, multiple business entities at different geographical locations may be involved within the process each using their own IT systems. As a result, good insights into the operation of the entire business process are missing. This causes that before a new IT is introduced it is vital that its impact on the entire business process is assessed. Therefore, in this paper, a process-oriented methodology is proposed for evaluating the impact of IT. In this method, both process mining and discrete event simulation are key ingredients. Process mining aims at extracting process knowledge from so-called event logs (6). Such logs may originate from all kinds of systems, such as generic enterprise information systems, product data management systems, or middleware systems. Typically, event logs contain information about the start and completion of process steps, along with related context data (e.g. actors and resources). Since process mining uses factual execution data it allows for obtaining an objective view on how processes are really executed. Moreover, it allows for obtaining quantitative insights into these processes (e.g. performance information). By means of discrete event simulation, the performance of systems can be evaluated, alternative configurations of a system can be compared, and an optimal configuration of a system can be found (7). In the context of a business process, a discrete event simulation model allows for accurately mimicing an existing business process. Next, the business process in the simulation model can be modified and the impact on selected performance indicators can be predicted.

In order to illustrate the above mentioned process-oriented methodology to evaluate the impact of a technology, a detailed case study is presented. In this study the effects of digital dentistry on the implant value chain is investigated using process mining and discrete event simulation. The implant value chain is concerned with all steps that can be associated with dental implants, covering the stages from patient diagnosis until implant placement. Key players in this value chain are the dentist (General Practitioner or GP), dental surgeon, and the 
dental laboratory. While it is expected that the application of IT, referred to as digital dentistry, improves the efficiency of the overall value chain and decreases the time that elapses from diagnosis until placement (8), the extent of this effect is unknown. That is, for the usage of digital techniques such as CAD/CAM for the design and production of the dental restoration; an Intra-Oral Scanner (IOS) for making a digital impression of a patient's teeth; and guided surgery for the planning and the guided placement of implants, it is anticipated that they each contribute in reducing the overall treatment time but that they also contribute in reducing the time needed by both dentists and dental lab technicians for performing the tasks in the business process. However, the exact impact of these three techniques on a business process is not known yet. The application of our technology shows how the impact of above mentioned technologies can be quantified.

All together, this paper addresses two different subjects. As a research subject, a processoriented methodology, using process mining and discrete event simulation, is proposed for investigating the impact of IT. Here, a core point of our methodology is that it can be applied to complex and distributed business processes (i.e. multiple business entities and various IT systems) for which it is hard to investigate new aspects. From a practical point of view, the impact of digital dentistry is investigated using the latter mentioned methodology. To date, we are not aware of any methodology for evaluating the impact of a new technology which uses both process mining and discrete event simulation. Various methods exist for evaluating the impact of new technologies but none of these focus on assessing the impact on an entire business process using both process mining and simulation.

The remainder of this paper is organized as follows. Section 2 discussed related work. In Section 3 , the process-oriented methodology for evaluating the impact of IT is presented.

Subsequently, in Section 4, this method is illustrated in the context of an extensive dental case. In Section 5, a reflection on the proposed methodology and the practical outcomes of the dental case is provided. Finally, a conclusion and outlook are provided in Section 6.

\section{Related Work}

For business processes, simulation has already been in use since the seventies (9). Before that, in the sixties, the simulation language SIMULA was developed which influenced the development of general purpose programming languages (10). Initially, the focus of these languages was on general purpose programming. However, these were subsequently extended with simulation capabilities. Gradually more and more simulation packages became available that offered some graphical environment to design and simulate business processes. These 
languages provide simulation building blocks that can be composed graphically (e.g. Arena (11)). Furthermore, most business process modeling tools provide some form of simulation. Examples of these tools are Protos and ARIS. Finally, the more mature Workflow Management Systems provide simulation capabilities for the processes they support (12;13) (cf. FileNet (14), BPM|One (15), BizFlow (16), and WebSphere (17)).

With respect to the application of simulation in the healthcare domain, numerous studies can be found reporting on the successful application of discrete-event simulation in order to improve efficiency and reduce costs. Several review papers have been written on the conduct of simulation studies in healthcare clinics (18) showing its wide spread use in this area including laboratory studies, emergency services, and the national health system. Good overviews of literature have been provided by Jun et al. (18), England et al. (19), and Yang et al. (20). With regard to healthcare clinics, Jun et al. (18) mention three different areas that impact patients in clinics. These are patient scheduling and admissions, patient routing and flow schemes, and scheduling and availability of resources.

However, despite the abundance of literature that exists on simulation and its application, there is hardly any literature on the intersection of process mining and discrete-event simulation. That is, we are only aware of the work of Rozinat et al. (21) in which process mining is used for building a discrete-event model. In particular, the aim of their work is to demonstrate that various perspectives of an existing business process (e.g. the control-flow, data, and resource perspective) can be discovered using process mining and that they can be stitched together in one simulation model. In other words, no details are provided regarding the evaluation of new technologies. Furthermore, there are some approaches in which a methodology is proposed for the application of process mining. That is, Bozkaya et al. (22) propose a quick-scan methodology in which several perspectives of a business process are discovered; based on the method of Bozkaya et al., Rebuge and Ferreira (23) propose a methodology in order to apply process mining in the healthcare domain; and Van der Aalst (6) proposes the $L^{*}$ life-cycle model in which the life-cycle of a typical process mining project is described using five stages. Related to this is the work of Zhou and Piramuthu (24) in which a framework is proposed in which a running business process is continuously optimized using process mining. The above mentioned methodologies all focus on the analysis of an existing business process. They do not aim on evaluating a change within these processes.

With regard to evaluation of Information Systems (ISs), there is a lot of debate within literature. For IS evaluation, Marthandan and Tang indicate that this is a complex task and that there is no common agreement on how best to do the evaluation as well as the selection of the 
appropriate evaluation criteria (25). Furthermore, IS evaluation is difficult as stakeholder perspectives may be different (26). As a consequence, Marthadon et al. indicate that there is no single method that works best to define and measure success (25). In general, IS evaluation is guided by several approaches (25). One approach concerns an ex ante or ex post evaluation. An ex ante evaluation focuses on project feasibility and is performed before an investment decision is made. An ex post evaluation examines the business value of the IT and analyzes if expected benefits have been realized. Another approach concerns a summative evaluation versus a formative evaluation. A formative evaluation is an ongoing evaluation during development which influences the attributes and features of the final IS. A summative evaluation is performed after implementation in order to assess the impacts the system has brought. Finally, the last approach concerns the usage of quantitative or qualitative methods during the IS evaluation. Some examples of well referred IS evaluation models (27) are the DeLone and McLean IS success model in which six different dimensions are provided in order to measure the success of IS (5), Grover et al. (4) distinguish between the organization and individual level in order to classify IS measures, Cronk and Fitzgerald (28) propose three dimensions of "IS business value": the system dependent dimension; the user dependent dimension; and the business dependent dimension, Seddon et al (3) propose to classify IS effectiveness measures according to a two-dimensional matrix which distinguishes between the stakeholders of the system and the type of system studied, and Smithson and Hirschheim (29) present a literature framework for analysis and IS evaluation. For the above mentioned IS evaluation models it holds that the measures contained in them still need to be materialized. As a result, they cannot immediately be used for evaluating the impact of IT.

Also, within the healthcare domain it is identified that the evaluation of Health Information Systems (HIS) is a complex task (30), in particular it is hard to select the framework to be applied and methods to be used (31). Yosof et al. (31) and Ammenwerth et al. (30) provide a good elaboration on the problems that need to be tackled. Furthermore, Yusof et al. (31) review a number of frameworks in order to identify the evaluation dimensions and measures used to evaluate systems in a healthcare setting. Both for the IS evaluation frameworks and the HIS evaluation frameworks it holds that none of them focus specifically on processes. Even more, no qualitative insights are provided regarding the effects of IT on the entire supply chain which consists of multiple distributed actors. Also, for none of the them, process mining and discrete event simulation are both used. 


\section{Process-oriented Methodology for Evaluating the Impact of IT}

In this section, we focus on the process-oriented methodology for evaluating the impact of IT. Core point of our methodology is that it can be applied to complex and distributed business processes (i.e. multiple business entities and various IT systems) for which it is hard to investigate new aspects. As in this methodology, both process mining and discrete event are key ingredients; they both will be briefly introduced. Afterwards, the methodology itself will be discussed.

\section{Process Mining and Discrete Event Simulation}

Process mining is applicable to a wide range of systems. The only requirement is that the system produces event logs, thus recording (parts of) the actual behavior. For these event logs it is important that each event refers to a well-defined step in the process (e.g. a lab test) and is related to a particular case (e.g. a patient) (32). Also, additional information such as the performer of the event (i.e. the doctor performing the test), the timestamp of the event, or data elements recorded along with the event (e.g. the age of the patient) may be stored. Based on these event logs, the goal of process mining is to extract process knowledge (e.g. process models) in order to discover, monitor, and improve real processes (6). Three types of process mining can be distinguished. The discovery type focuses on inferring process models that are able to reproduce the observed behavior. The inferred model may be a Petri net, a BPMN model, or an EPC. The conformance type aims at checking if observed behavior in the event log conforms to a given model (e.g a rule, a description of the current process). Finally, for the extension type, information extracted from the log is projected onto the model (e.g. performance information is projected on a discovered process model).

Discrete event simulation concerns the modeling of a system as it evolves over time by a representation in which the state variables change instantaneously at separate points in time (33). In the context of a business process, a discrete event simulation model allows for accurately mimicing an existing business process. Based on an existing business process, redesigns of it can be explored and evaluated before they are actually implemented. In the methodology that will be presented below, by using process mining, a simulation model can be (partly) generated based on process information extracted from event logs. In this way, the actual simulation phase can be started much quicker compared to the traditional approach, where simulation models are created manually. 


\section{Methodology}

In Figure 1, the process-oriented methodology for investigating the impact of IT, is shown. As can be seen, the methodology consists of both an as-is phase and a to-be phase. These two phases are the main axes in order to create a baseline for comparison.

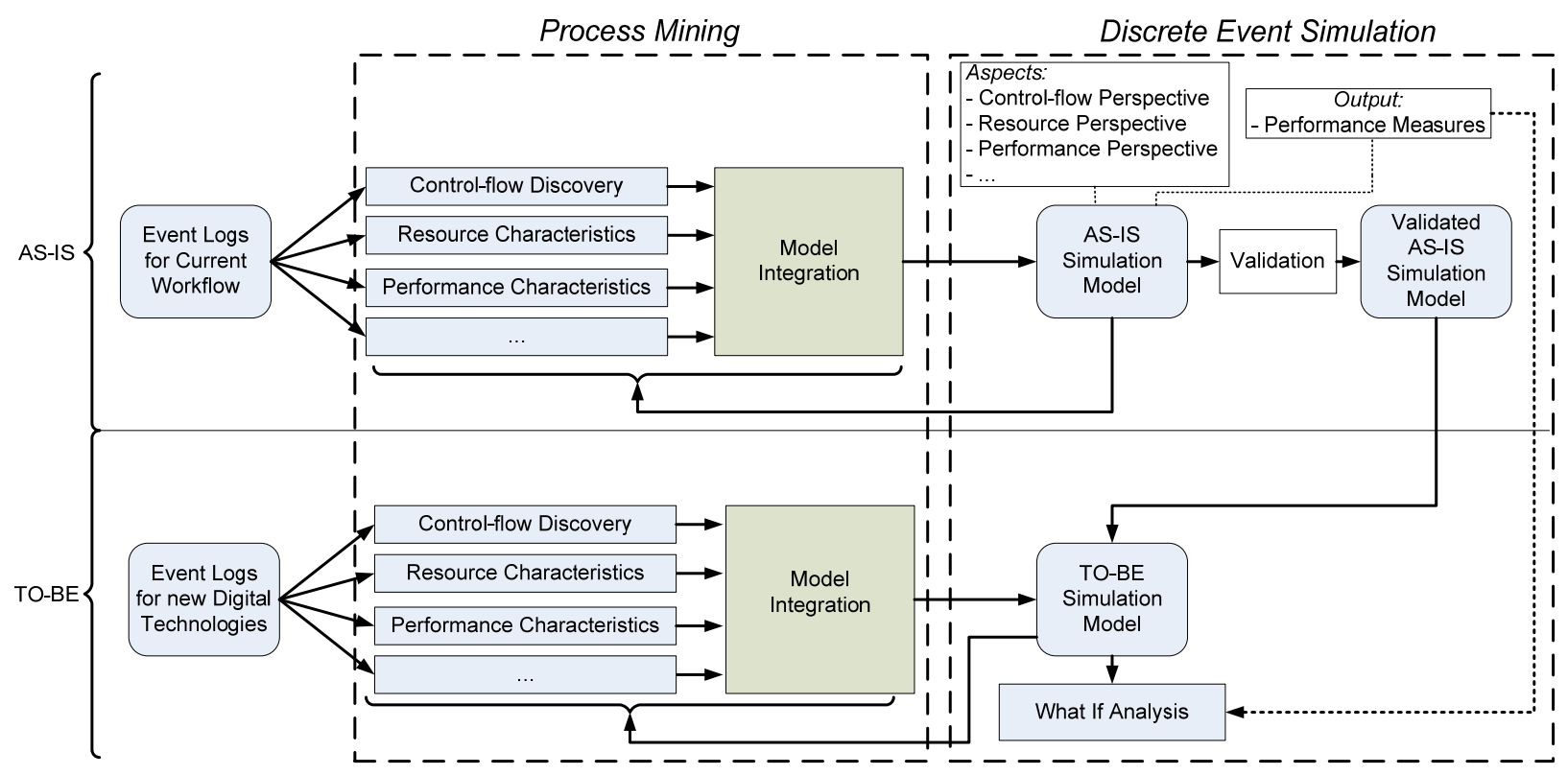

Figure 1: The process-oriented methodology for evaluating the impact of IT. In this methodology, process mining and discrete-event simulation are both used.

Starting point of the approach is that, in the AS-IS phase, event logs exist that describe the behavior of an existing business process. Afterwards, a combination of process mining techniques is used in order to discover multiple perspectives of the existing business process under consideration. Here, the control-flow, resource, and performance perspectives are the most straightforward ones. The control-flow perspective covers the tasks and its order, the resource perspective covers the resources that are performing the tasks, as well as the performance perspective covers the timing of the tasks (e.g. execution time and waiting time) and the arrival pattern of new cases. Depending on the IT of which its impact needs to be assessed, other perspectives may need to be discovered. Afterwards, the obtained results for the chosen perspectives are stitched together in one model and a simulation model is generated. Next, the simulation model is validated in order to statistically verify that the generated model accurately mimics the behavior of the business process under consideration. For the validation, performance measures need to be defined within the AS-IS simulation model and multiple replications of the simulation model are needed in order to obtain reliable 
statistical results. For each performance measure, the average value that has been obtained for the multiple replications of the simulation model can be compared with the corresponding value that has been obtained by process mining. In this way, it can be determined whether the simulation model is valid. Note that in order to obtain a valid simulation model it may be needed to apply the process mining algorithms again or that even new algorithms need to be developed.

In the TO-BE phase, the business process in the validated simulation model is redesigned in order to investigate the impact of a new digital technology for that process. For this new technology, it is assumed that execution data for it is available in which the technology has been applied in a different context than for the redesigned process in the TO-BE phase. However, for the obtained execution data it is important that it is valid to include it in the redesigned process in the TO-BE phase. For example, assume that in the redesigned process we want to include technology ' $A$ '. For technology ' $A$ ' execution data is available in which it is used in a different context than the redesigned process. However, as for the redesigned process the context is comparable with the context in which technology ' $A$ ' is used, the execution data can be included in the redesigned process in the TO-BE phase. Next, using a combination of process mining techniques, the same perspectives as within the AS-IS phase are discovered. Subsequently, the perspectives are stitched together and integrated in the validated AS-IS simulation model such that a TO-BE simulation model is obtained which can be used for a 'what-if analysis'. In this 'what-if analysis', the impact of the new technology can be investigated based on the performance measures that have been included in the simulation model in the AS-IS phase. Note that for the TO-BE simulation model, it may also be needed to develop new process mining algorithms.

ProM has become the de facto standard for process mining. The ProM framework (www.processmining.org) is a 'plug-able' environment for process mining using MXML, SAMXML, or XES as input format. ProM 5.2 contains multiple plug-ins for the discovery of the control-flow, resource, and perspective perspectives. Furthermore, the results for multiple perspectives can be stitched together in one simulation model and exported to the CPN-tools simulation environment (21). In this paper, all presented process mining results are obtained using ProM 5.2.

\section{Case: the Impact of Digital Dentistry}

In this section, we focus on an extensive dental case in order to demonstrate the processoriented approach that has been presented in the previous section. So, the effects of digital 
dentistry on the implant value chain is investigated using process mining and discrete event simulation. The implant value chain is concerned with all steps that can be associated with dental implants, covering the stages from patient diagnosis until implant placement. Key players in this value chain are the dentist (General Practitioner or GP), dental surgeon and the dental laboratory. It is expected that the application of IT, referred to as digital dentistry, improves the efficiency of the overall value chain and decrease the time that elapses from diagnosis until placement. In the next subsections, the execution data that has been used, the simulation model that has been constructed, and the what-if analyses that have been performed, are elaborated upon.

\section{Data and Analysis}

As indicated before, one of the subjects of this paper is, from a practical point of view, to investigate the effects of digital dentistry on the implant value chain. Therefore, it is essential to have a detailed, quantitative understanding of all the steps in the current value chain - the "ASIS" situation. Using process mining, a view of the AS-IS chain is distilled, which consists of all the steps that are taken in the current situation (i.e. the control-flow perspective), the actors that are involved in carrying out these steps (i.e. the resource perspective), and their performance (i.e. the performance perspective). In order to have an overview of the current value chain, in the 'Current Value Chain' Section, the discovered chain will be discussed and analyzed.

Next to that, in order to investigate the effects of digital dentistry - the TO-BE situation, detailed execution data of different digital aids needs to be available. These digital aids will be presented and analyzed in the 'Digital Dentistry' Section.

\section{Current Value Chain}

In the current value chain, key players are the dentist, dental surgeon, and the dental laboratory. Dentistry is the branch of medicine that is involved in the study, diagnosis, prevention, and treatment of diseases, disorders and conditions of the oral cavity, the maxillofacial area and the adjacent and associated structures, and their impact on the human body (34). As such, dentistry is primarily focused on human teeth, although it is not limited strictly to this. In order to support restorations that resemble a tooth or a group of teeth (e.g. crowns, bridges, and dentures), one or more dental implants can be used. A dental implant is a 'root' device, usually made of titanium.

In order to arrive at a good understanding of the activities performed by the above mentioned key players within the entire value chain, we obtained execution data from the dental practices 'Wilhelminaweg' and 'Academic Centre for Dentistry Amsterdam'. The two dental practices 
collaborate intensively with the 'Dental Lab Zutphen'. Therefore, data from this dental lab also has been obtained in order to get a good understanding of the activities performed in a dental lab.

Dental Practice Wilhelminaweg: The dental practice Wilhelminaweg (http://www.tpwdieren.nl) is a medium sized private dental practice in the Netherlands. Here, several experienced dental specialists and implantologists are working. The dental practice has a clinical management system in which all appointments are saved that have taken place for a patient.

Academic Centre for Dentistry Amsterdam (ACTA): The ACTA is a collaborative venture involving the Faculties of Dentistry at the University of Amsterdam and VU University Amsterdam. In the department of Oral Implantology and Prosthodontics experienced implantologists are working as well as dentists that are in training in order to become an implantologist. For all patients that are treated, in the clinic management system, all the appointments and their timing are stored.

Dental Lab Zutphen: The Dental Lab Zutphen (http://www.ttlzutphen.nl) is a medium sized dental lab in the Netherlands. Amongst others, the lab takes care of the entire process of implant borne restorations such as crowns on implants and a prosthesis on implants. The lab has a dedicated lab management system in order to track all the products that are made in the lab. For each product that is made, it is stored which steps are performed and their timing. However, it needs to be mentioned that for the dental lab it is only recorded on which day a certain task is executed. This has its consequence on the timing information that is obtained for the tasks.

For investigating the impacts of digital dentistry, the 'prosthesis' and 'crown' business processes have been selected. These two business processes have been chosen because they are two of the most common applications of implants. Also, they are largely distinct from each other in terms of the activities that are performed and the approach that is followed for producing the final dental restoration. As such, it allows for investigating the impact of different digital dentistry techniques. The process mining results of the two business processes are discussed below. In a similar fashion as the methodology proposed in Figure 1, for each business process, the results that have been obtained for the control-flow, performance, and resource perspective will be presented respectively. Note that both business processes have been discovered based upon data of the three companies discussed above. 


\section{Prosthesis Process}

The process mining results for the control-flow, resource, and performance perspective of the 'prosthesis' business process are visualized in Figure 2. The model shown in the figure is a High Level Petri net (35); rectangles represent tasks, double-lined rectangles represent composite tasks, and circles, called places, represent a state in the process. Note that fully black rectangles are only added to accurately describe the flow of work. A composite task models a subprocess consisting of multiple tasks. Furthermore, the color of a place provides an indication about the average waiting time that is spent in the place; a pink color indicates a high waiting time (more than 60 days), a yellow color indicates a medium waiting time (in between 30 and 60 days), and a blue color indicates a low waiting time (less than 30 days). Furthermore, several places have multiple outgoing arcs. This represents a choice in the process and only one path may be followed. For each outgoing arc, the probability of following the respective path is indicated. Note that the prefix of a task indicates the role that needs to be played by the person which is allowed to perform the task. Here, the 'dentist' role is represented by a ' $D$ ', the 'dental surgeon' role is represented by a 'DS' and the 'lab' role is represented by an ' $\mathrm{L}$ '.

The control-flow and the roles for the resource perspective are as follows. First, there is an implant consultation (task "D: implant consultation") in which the patient and the dentist discuss the placing of an implant. Afterwards, several steps may occur before the placing of implants which is done by a dentist (task "D: place implants") via means of free-hand drilling. For example, there is an average probability of ' 0.06 ' that the dentist and the patient discuss the treatment plan (task "D:discuss treatment plan") and there is an average probability of '0.09' that the dentist decides that an additional consultation is needed (task "D:consultation"). Also, it may be required to perform a couple of checks before the start of the treatment (task "D:checklist") or to evaluate the treatment plan (task "D:evaluation"). After the placing of the implants, several steps may be performed. This may involve a consultation ("consultation" task), a check-up of the implants ("check-up" task), or an adjustment of the prosthesis ("adjust prosthesis" task). For the latter two steps, this may also be done during a single appointment ("adjust prosthesis + check-up" task). Furthermore, a series of steps may be performed in order to produce the final prosthesis which requires the involvement of the dentist and the dental lab. Note that in order to not clutter the model the steps of the lab are represented by composite tasks. First, dental impressions are made by a dentist ("D:first impressions" task) followed by the making of individual impression trays by the lab (L:make individual impression trays task). Afterwards, individual dental impressions are made by the dentist (D:individual impressions task). In turn, the lab makes a plate ("L:prepare registration bite" task) such that afterwards the bite of the patient can be registered by the dentist ("L:prepare registration bite" 
task). Next, the lab and the dentist collaborate in order to arrive at a fitting prosthesis. The lab makes a wax model of the prosthesis ("L:prepare for fit" task) which the dentist fits in the mouth of the patient. The fitting of the prosthesis can be divided into the fitting of the entire prosthesis ("D:fit prosthesis" task), the fitting of the front teeth ("D:fit prosthesis (front)" task), or the fitting of the molars ("D:fit prosthesis (molars)" task). Once the patient is fine with the prosthesis, it is finalized by the lab, using the lost-wax method, and subsequently the prosthesis is placed in the jaw ("D:place prosthesis" task).

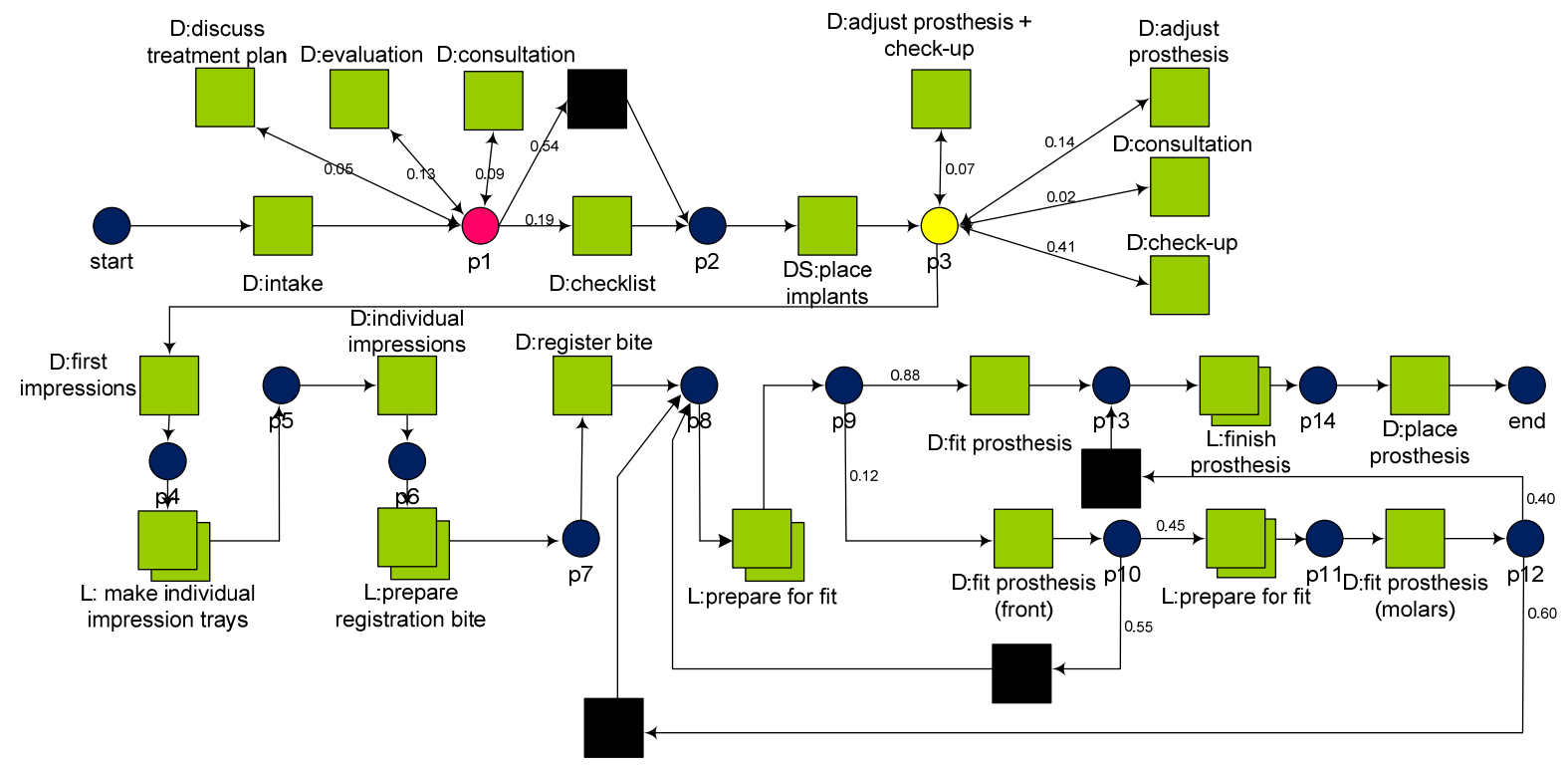

Figure 2: 'Prosthesis' business process in the AS-IS situation. The prefix of a task indicates whether the step is performed by a dentist (D), a dental surgeon (DS), or the dental lab (L).

For the performance perspective it can be seen in Figure 2 that there is a high waiting time after the intake. That is, in the place called ' $p 1$ ', the average waiting time is 87.11 days (standard deviation: 74.8 days). This is mostly due to the fact that an acceptance from the health insurance agency needs to be obtained. Furthermore, also after the placing of the implants there is quite some waiting time. In place 'p3', the average waiting time is 43.9 days (standard deviation: 21.1 days). This is due to the fact that after the surgery, considerable time is needed for the healing of the wound. Furthermore, it is noticeable that the average times it takes to move work from the dentist to the lab and the other way around, are low (on average 5 days). This is because a dentist indicates to the lab the date of the next appointment with the patient. In this way, the lab has the flexibility to arrange the work such that just before the next patient appointment the dental lab product is delivered to the dental practice. However, it 
should be noted that on average the lab has to finish its work within 2 weeks. Furthermore, the production of the final prosthesis requires at least 4 patient appointments and at least 4 times that work is performed by the lab.

Additionally, for the resource perspective, it has been found that, especially for ACTA, many different dentists are involved in the entire treatment of patients. This can be seen in Figure 3 which shows for a selected number of patients a dotted chart (36). In this chart for each patient the subsequent tasks performed by a dentist are shown. The color of a dot visualizes the dentist which has performed the task. As can be seen, there are patients that always see the same dentist but there are also patients that see many different dentists. For example, the patient at the top of the figure has had 4 appointments and 2 different dentists whereas the patient at the bottom of the figure has had 19 appointments and 6 different dentists. Also, but not visible in the figure, dentists may be experienced or not. This has its impact on the duration of the tasks and the tasks that are performed in the business process. For example, it has been found that inexperienced dentists have a strong preference for splitting the fitting of the prosthesis into multiple appointments, such as fitting the front teeth and the molars.

Based on the above presented results for the three perspectives, it would be worthwhile to investigate redesigns in which digital technologies facilitate in reducing the work that needs to be done by the dental lab and the dentist for the production of the prosthesis. Regarding the healing time after the placing of the implants, it would be interesting to investigate a redesign in which the healing time is not part anymore of the entire business process, i.e. the implants and the final restoration are both placed at the end of the business process. As such, the total throughput time of the entire process is reduced. For the resource perspective, it is important that the execution of tasks by either inexperienced or experienced dentists are carefully captured in the simulation model in order to guarantee that the model matches reality as close 
as possible.

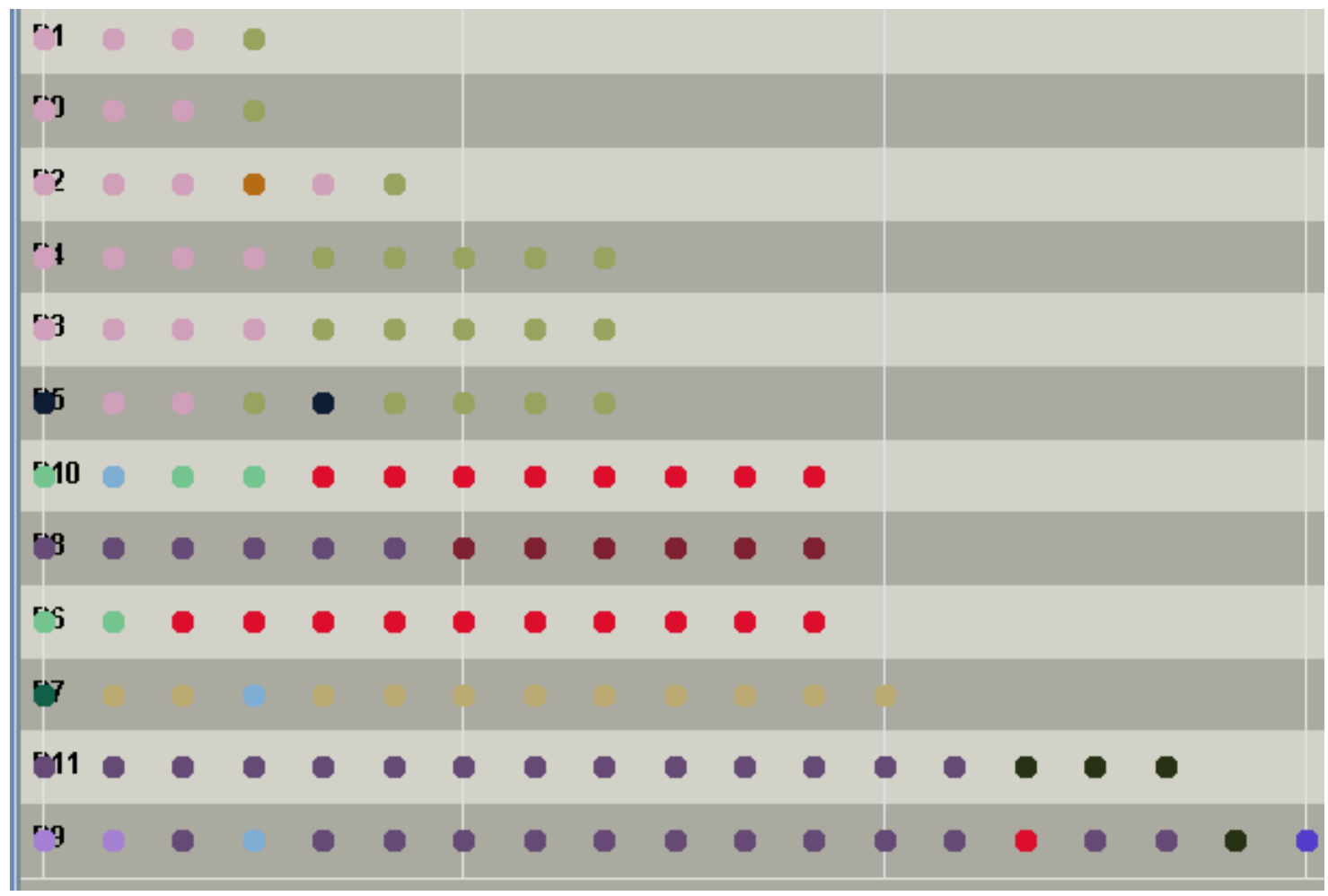

Figure 3: Dotted chart showing for each patient all the subsequent tasks that have taken place. The color of a dot visualizes the dentist which has performed the task.

\section{Crown Business Process}

The process mining results for the control-flow, resource, and performance perspective of the 'prosthesis' business process are visualized in Figure 4. The control-flow and the roles for the resource perspective are as follows: First an intake takes place which is done by a dentist ("D:intake" task). Before the placing of the implants ("D:place implants" task) via free-hand drilling, there is the option to have a consultation ("D:consultation" task), to discuss the treatment plan ("D:discuss treatment plan" task), to extract a tooth ("D:extraction" task), to perform a check ("D:check" task), to check points from a checklist ("D:checklist" task), or to do the latter in combination with making study models ("D:checklist + study models" task). Furthermore, the dental hygiene is checked ("D/H:Hygiene" task) which is done either by a dentist or a dental hygienist. After the placing of the implants it is possible to have a check-up ("D:check-up" task), to check the dental hygiene ("D/H:hygiene" task), or to expose the implants ("D:expose implants" task). During the latter three steps it is possible that the dentist makes an dental impression that can be used by the dental lab for making individual impression trays (“L:make individual impression trays" task). Using this individual impression tray, an 
individual dental impression is made ("D:make impression" task). Subsequently, by using the lost-wax method, the dental lab makes the final crown ("L:make crowns" task) which is afterwards placed in the mouth of the patient ("D:place crown" task).

Regarding the performance and resource perspective, we experienced a similar situation as for the 'prosthesis' business process. However, it is noticeable that there is a medium waiting time after the surgery. This is due to the fact that after the surgery, considerable time is needed for the healing of the wound.

Finally, for the 'crown' business process it is worthwhile to investigate redesigns in which digital technologies facilitate in lowering the throughput time of the entire process but also the time needed by the dentists to perform the tasks in the entire process.

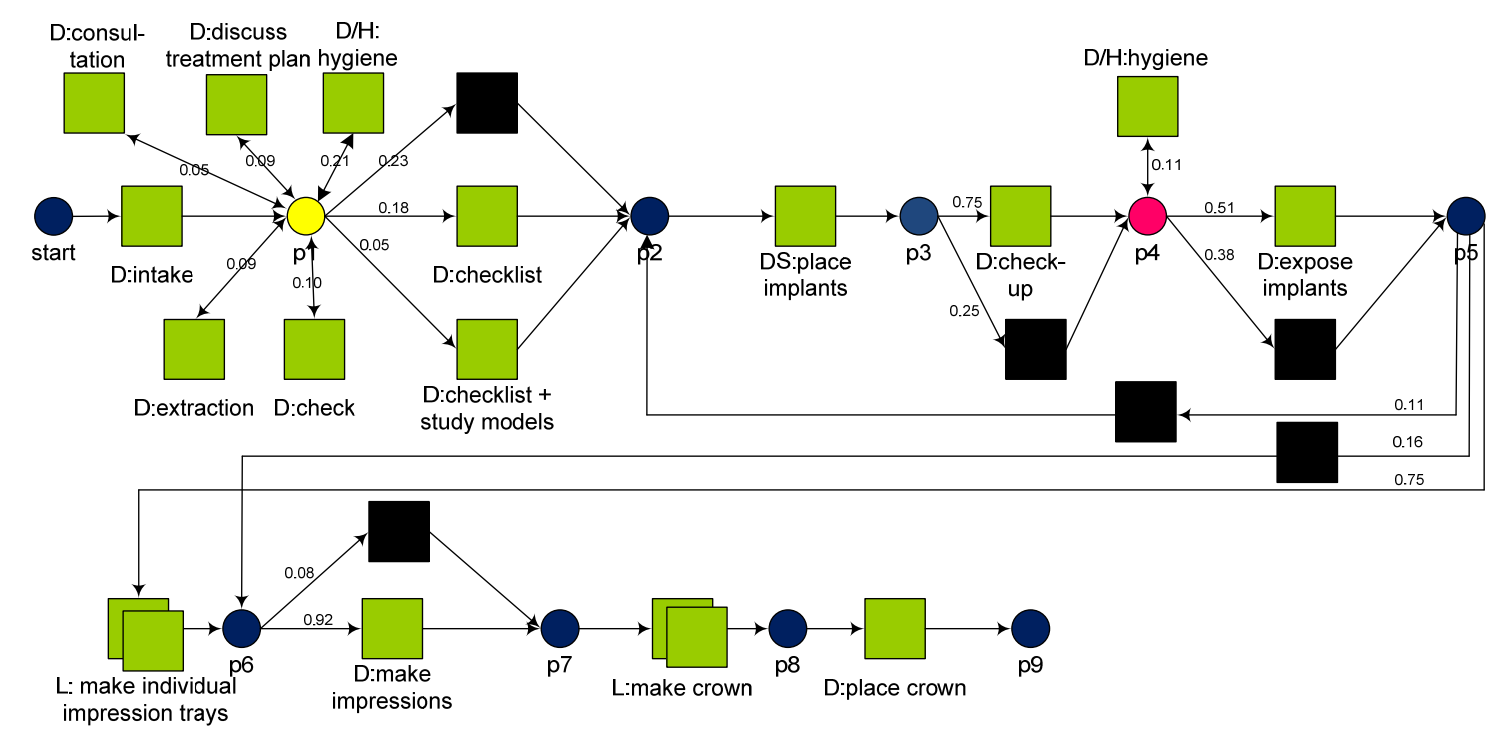

Figure 4: Crown business process in the AS-IS situation. The prefix of a task indicates whether the step is performed by a dentist (D), a dental surgeon (DS), a dental hygienist (H), or the dental lab (L). Furthermore, a pink-colored place indicates a high waiting time (more than 60 days), a yellow-colored place indicates a medium waiting time (in between 30 and 60 days), and a blue-colored place indicates a low waiting time (less than 30 days).

\section{Digital Dentistry}

For both the prosthesis and crown business process it became clear that the introduction of new IT should facilitate in lowering the throughput time of the entire process and lowering the total working time of both the dentists and the lab technicians. Based on interviews with dental experts we have identified for both the 'prosthesis' and the 'crown' business process, three 
conventional technologies that are used in these processes and which are important candidates for being replaced by new IT technologies. By introducing one or more new IT technologies in a business process, it is anticipated that the above mentioned reductions can be realized.

That is, at the top of Table 1 (caption 'AS-IS'), it is shown that for both the 'prosthesis' and the 'crown' business process, the dental impression is made using an impression tray, the implantation is done using free-hand drilling, and that the production of the restoration is done using conventional techniques (e.g. the lost-wax method).

Table 1: At the top of the table (caption 'AS-IS'), for the current 'prosthesis' and the current 'crown' business process it is shown which techniques are used within the process. At the bottom of the table (caption 'TO-BE') for redesigns of the prosthesis and the crown business processes, it is indicated which new technologies are used for the dental impression, implantation, and the production of the restoration.

\begin{tabular}{|c|c|c|c|c|c|c|}
\hline \multicolumn{7}{|c|}{ AS-IS } \\
\hline & \multicolumn{6}{|c|}{ Technique } \\
\hline & \multicolumn{3}{|c|}{ Conventional } & \multicolumn{3}{|c|}{ Digital } \\
\hline & $\begin{array}{l}\quad \text { Dental } \\
\text { impression: } \\
\text { Impression } \\
\text { tray }\end{array}$ & $\begin{array}{l}\text { Implantation: } \\
\text { Conventional } \\
\text { free-hand } \\
\text { drilling }\end{array}$ & $\begin{array}{l}\text { Production } \\
\text { restoration: } \\
\text { Conventional } \\
\text { techniques }\end{array}$ & $\begin{array}{l}\frac{\text { Dental }}{\text { impression: }} \\
\text { Intra-Oral } \\
\text { Scan (IOS) }\end{array}$ & $\begin{array}{l}\text { Implantation: } \\
\text { Guided } \\
\text { surgery (with } \\
3 \text { mini- } \\
\text { implants) }\end{array}$ & $\begin{array}{l}\frac{\text { Production }}{\text { restoration: }} \\
\text { CAD/CAM }\end{array}$ \\
\hline Prosthesis & $x$ & $x$ & $x$ & & & \\
\hline Crown & $x$ & $x$ & $x$ & & & \\
\hline \multicolumn{7}{|c|}{ TO-BE } \\
\hline & \multicolumn{6}{|c|}{ Technique } \\
\hline & \multicolumn{3}{|c|}{ Conventional } & \multicolumn{3}{|c|}{ Digital } \\
\hline & $\begin{array}{l}\frac{\text { Dental }}{\text { impression: }} \\
\begin{array}{l}\text { Impression } \\
\text { tray }\end{array}\end{array}$ & $\begin{array}{l}\text { Implantation: } \\
\text { Conventional } \\
\text { free-hand } \\
\text { drilling }\end{array}$ & $\begin{array}{l}\text { Production } \\
\text { restoration: } \\
\text { Conventional } \\
\text { techniques }\end{array}$ & $\begin{array}{c}\frac{\text { Dental }}{\text { impression: }} \\
\text { Intra-Oral } \\
\text { Scan (IOS) }\end{array}$ & $\begin{array}{l}\text { Implantation: } \\
\text { Guided } \\
\text { surgery (with } \\
3 \text { mini- } \\
\text { implants) }\end{array}$ & $\begin{array}{l}\frac{\text { Production }}{\text { restoration: }} \\
\text { CAD/CAM }\end{array}$ \\
\hline $\begin{array}{c}\text { Redesign 1: } \\
\text { Prosthesis }\end{array}$ & & $x$ & & $x$ & & $x$ \\
\hline $\begin{array}{c}\text { Redesign 2: } \\
\text { Prosthesis }\end{array}$ & $x$ & & & & $x$ & $x$ \\
\hline $\begin{array}{c}\text { Redesign 3: } \\
\text { Crown }\end{array}$ & & $x$ & & $x$ & & $x$ \\
\hline
\end{tabular}


For the 'TO-BE' situation, the interviews with dental experts made clear that the making of the impression can be done digitally using Intra-Oral Scanning (IOS), the placement of implants can be done using guided surgery, and that the design and the production of the dental restoration can be done using CAD/CAM. In Table 1, it is shown for two redesigns of the 'prosthesis' businesses process and one redesign of the 'crown' business process, which new technologies can be applied according to the interviewed dentists. For example, for the first redesign of the 'prosthesis' workflow, the dental impression is made using IOS, and the production of the restoration is done using CAD/CAM techniques.

Clearly, for the redesigned business processes, always two new technologies are introduced. Currently, each combination is not yet used in any respective business process but they are expected to become into use in the coming 5 years. The expectation was that these advances significantly improve the efficiency of the overall business process, decrease the time that elapses from diagnosis until placement, and help to increase the precision of placed implants. Furthermore, it was also expected that the new digital technologies can facilitate in realizing these redesigns.

Each of the proposed redesigns will be discussed later in more detail in the paper in order to evaluate the impact of digital dentistry. First, for the new technologies, shown in Table 1, detailed execution data was obtained. Each of them will be presented and analyzed below. In particular, in line with the methodology proposed in Figure 1, for each digital technology, the results that have been obtained for the control-flow, performance, and resource perspective will be presented respectively.

\section{Digital Design of Crowns and Bridges}

In the dental lab, a variety of products (e.g. the understructure of a crown, a full anatomic crown, and a multiple-unit bridge) are manufactured or customized in order to assist in the provision of oral health care by a dentist. For many years, these products have been made by hand using well established conventional techniques (e.g. the lost-wax casting technique). In order to increase precision and to reduce production time, several digital techniques have been introduced. One of these techniques is that (parts of) products can be designed and manufactured using CAD/CAM techniques (37).

A company allowing for designing an extensive range of prosthetic products is DentalWings (see http://www.dentalwings.com). Their software, called DWOS, is currently in use in many dental labs across various countries. One of their customers is the Dentalcam dental lab which is 
located in the Netherlands (http://www.dentalcam.nl). From this dental lab, execution data has been obtained. Some of the obtained results are the following.

In Figure 5, the control-flow, resource, and performance perspective of the business process followed for designing 751 products is shown. The control-flow and the roles for the resource perspective are as follows. Note that a person which uses the DWOS software is represented by the 'user' role. In the figure, the 'user' role is represented by the ' $U$ ' prefix. First, for an indication, an order is created ("U:Creation and First Initialization" task). Afterwards, several steps can be done. Note, that in order to perform a certain step, the order first needs to be locked ("U:Locked" task). Afterwards, the order is unlocked again (“U:Unlocked" task). After creation of the order, the model of the patient's teeth can be scanned ("U:Scan Done" task) or a file of a previous scan can be imported ("U:Scan Import Done" task). Next, there is the option to let the CAD Engine make a proposal for the design of the indication ("U:CAD Engine done" task). Within the DWOS software, this always needs to be triggered by a user. Afterwards, the last step is to complete the design ("U:Design Done" task). 


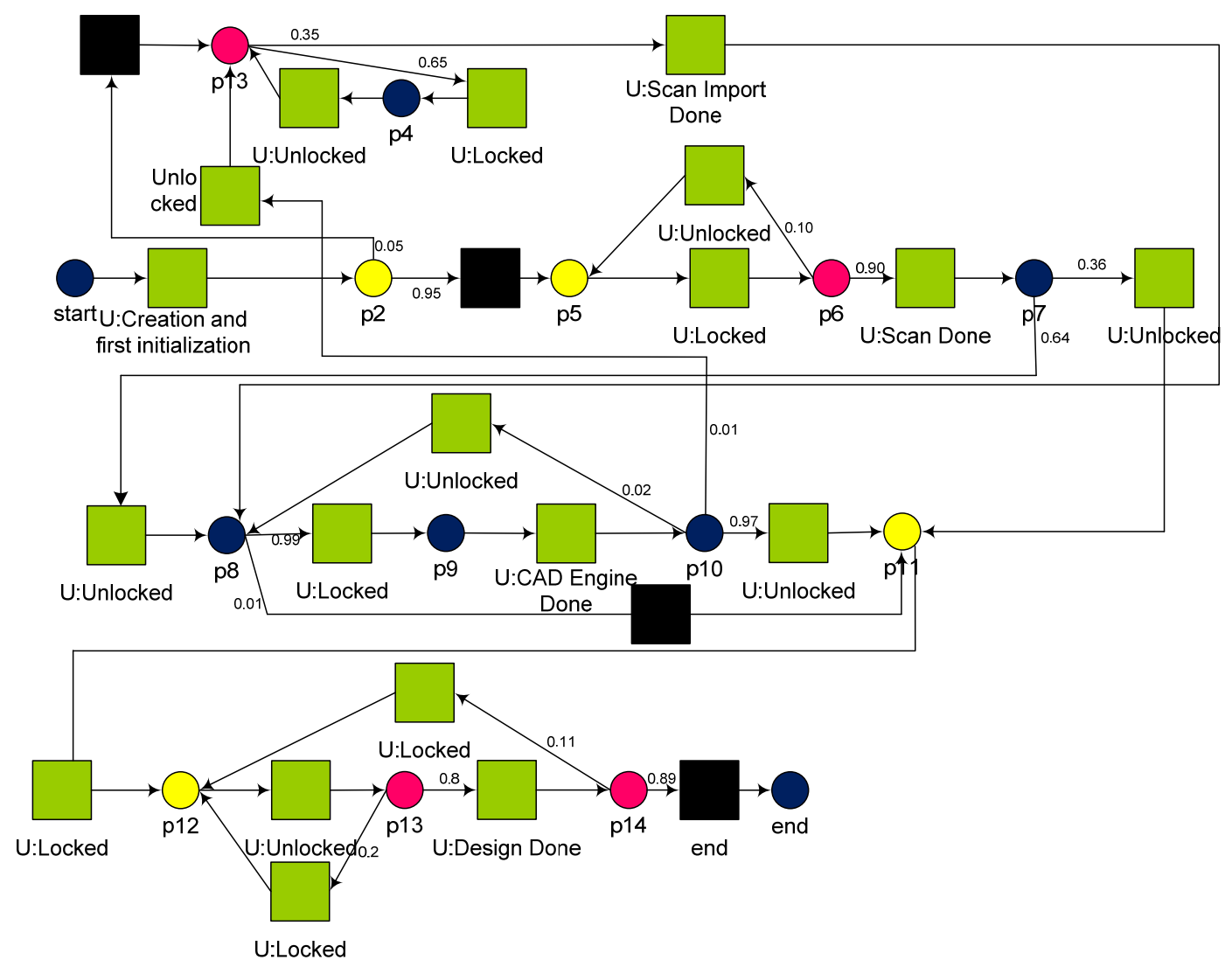

Figure 5: Business process showing how the Dental lab technicians used the DWOS CAD/CAM software for designing various dental products (e.g. a crown or a bridge).

Regarding the performance perspective it can be seen that there a couple of bottlenecks in the process (the pink colored places). A pink colored place indicates that a high waiting time exists for the place (more than 60 minutes), a yellow color indicates a medium waiting time (in between 30 and 60 minutes), and a blue color indicates a low waiting time (less than 30 minutes). These bottlenecks are all related to unlocking an order to continue working on a specific step. For example, in place "p13" on average 69 minutes (standard deviation: 620 minutes) is spent before locking the order again or to announce the completion of the design. Also, detailed information has been obtained about the time needed to complete a certain step. For example, it takes on average 6.8 minutes to design a three unit bridge (standard deviation: 4.31 minutes) and 2.96 minutes to design a single coping (standard deviation: 1.96 minutes). 
For the resource perspective it is important to mention, that for the execution data of the Dentalcam dental lab, it has been registered that all tasks shown in Figure 5 are done by the same user. This is due to the fact that within the DWOS software of Dentalcam only one user has been registered which is allowed to work with the software. However, we believe that still representative user behavior is obtained that can be incorporated in the simulation model to investigate the impacts of digital dentistry.

\section{Intra-Oral Scanning}

Intra-oral scanning is a relatively new technology that uses a low power laser to measure the position of the tooth as well as the artifact. The intra-oral scanner makes the traditional impression superfluous (38). So, instead of multiple appointments in which an impression needs to be made using an impression tray and putty it is also possible to have one appointment in which a digital impression is made using an Intra-Oral Scanner (IOS). Regarding the discovery of information for the control-flow, resource, and performance perspective, it was only possible that a selected dentist collected information about the time that was needed for making a digital impression for a group of 17 patients. As a result, for the control-flow and resource perspective only a single task of making a digital impression for a patient has been carried out which is always done by the same dentist. Regarding the performance perspective, 
the bar chart in

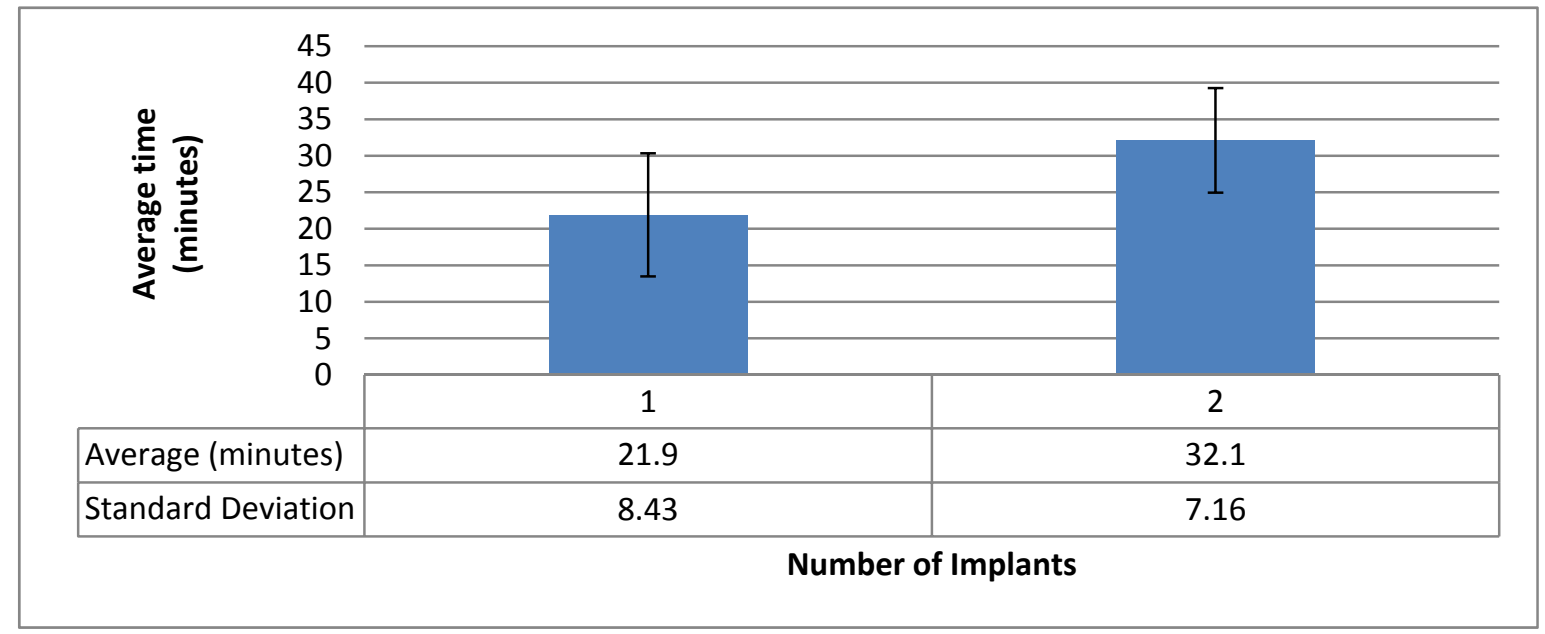

Figure 6 shows the average time needed for the IOS of one and two implants. For example, it takes on average 22 minutes to scan a mouth with one implant and 33 minutes to scan a mouth with two implants. Note that the above given figures for making an IOS are comparable to the time that is needed for making an impression using an impression tray.

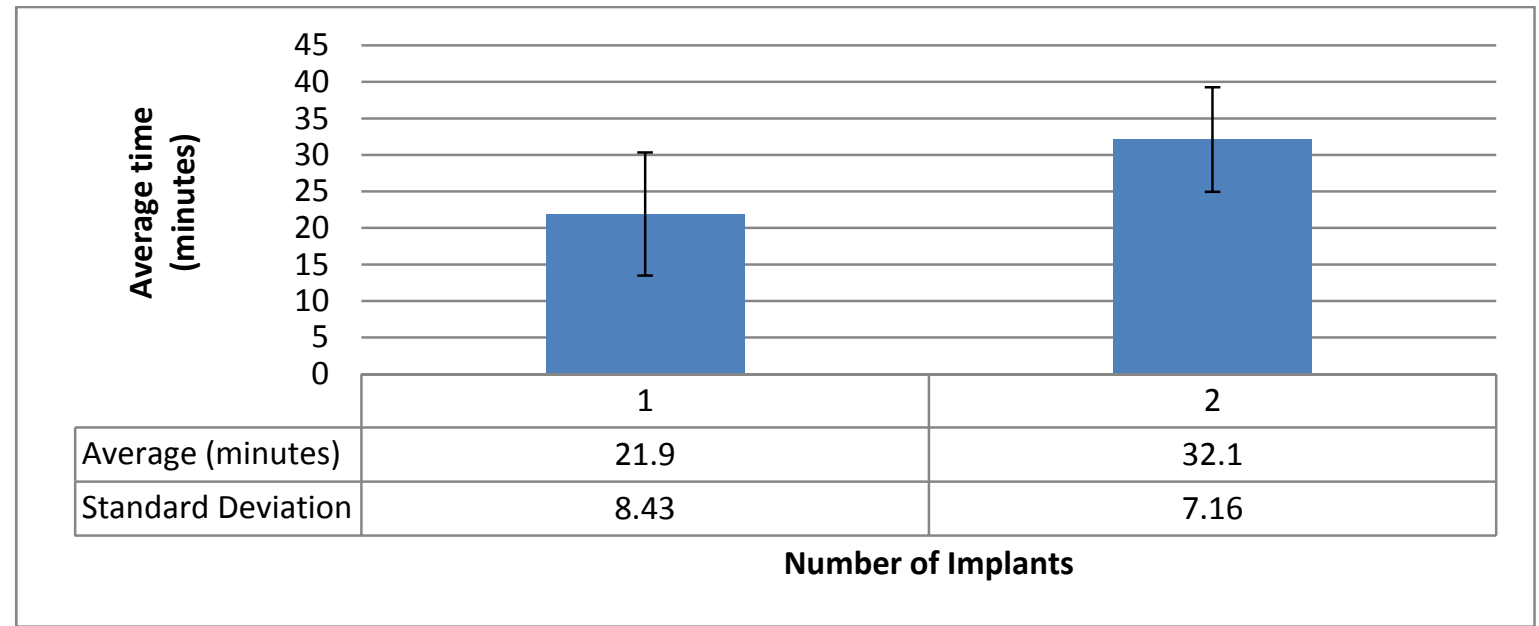

Figure 6: Bar chart depicting the average time needed for the IOS of one and two implants. Furthermore, the associated error bars are shown (representing one standard deviation).

\section{Guided Surgery}

Typically, implants are placed using a mechanical hand drill. Using guided surgery, a dedicated software program allows for 3D diagnostics and implant planning. After the planning of the implants, a drilling guide can be made such that the implants are placed in the same position as 
planned in the software program (39). A software program that allows for the planning of implants and that afterwards the drilling guide can be produced is CoDiagnostiX (http://www.codiagnostix.com). The advantages of guided surgery compared to the conventional method is that the surgery is better controlled as the guidance of surgical instruments allows the surgeon to perform the surgery exactly according to plan. Moreover, fewer replacements are required and the duration of the surgical intervention itself is considerably reduced. Currently, only few dentists are using guided surgery for the placement of implants as the planning software and the production of the drilling guide is quite expensive. Furthermore, dentists are hesitant to adopting the new technique.

For the CoDiagnostiX software program, for a group of 19 patients, execution data has been obtained about the time that a user started working on a presurgical planning and the time that a user completed the presurgical planning. So, for the control-flow and resource perspective, there is only a single task of making a presurgical planning for a patient which has always been done by the same person. Regarding the performance perspective, the bar chart in Figure 7 shows the average planning time per number of implants. For example, in total, on average 32 minutes are needed for 2 implants and 45 minutes for 6 implants. Furthermore, it seems that for 2, 3, and 4 implants the planning time is comparable whereas for 5 and 6 implants this is considerably higher.

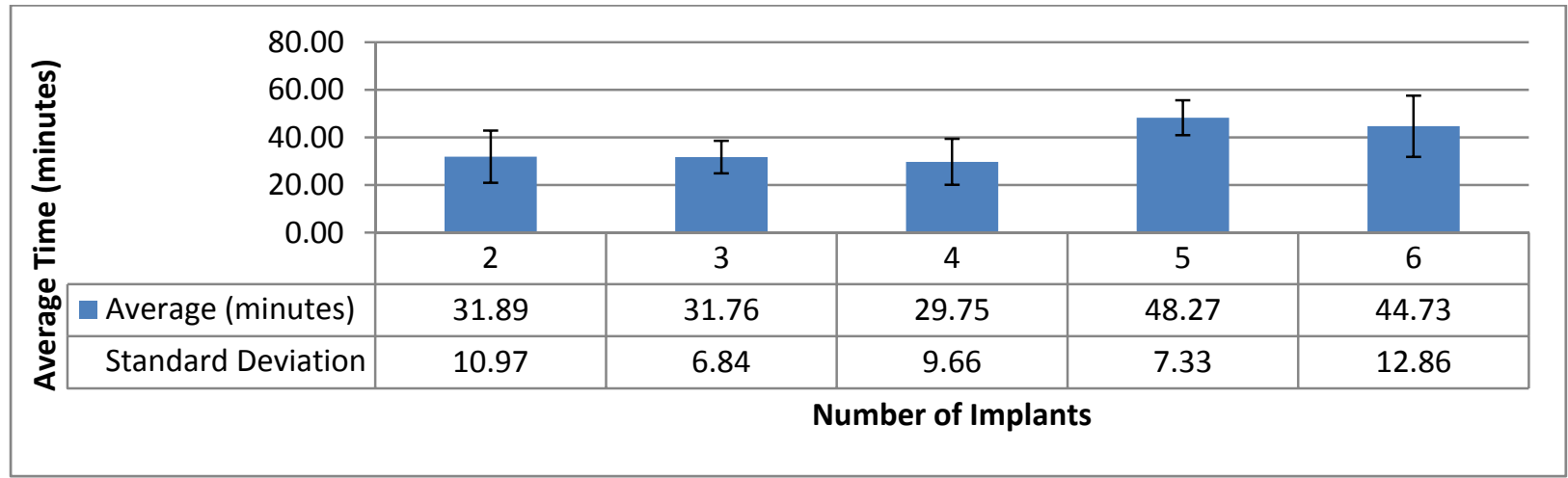

Figure 7: Bar chart depicting, for different numbers of implants, the average time needed for the planning of the guided surgery using the CoDiagnostiX software. Furthermore, the associated error bars are shown (representing one standard deviation).

\section{Simulation}

In order to investigate the impacts of digital dentistry, a quantitative simulation model will be built that captures the business process of placing a crown and the business process of placing a prosthesis (AS-IS). Subsequently, the simulation model is used for investigating the impact of 
digital dentistry. In this section, the simulation model will be discussed in detail. First, we elaborate on the building of the simulation model in Section 'Making the Simulation Model' followed by the validation of it in Section 'Validation'. Finally, several redesigns for investigating the TO-BE situation are described in Section 'Redesigned Business Processes'. That is for the 'prosthesis' business process, two redesigns will be discussed and for the 'crown' business process one redesign will be discussed. In these redesigns the new techniques presented in Table 1 will be used.

\section{Making the Simulation Model}

In this section, the most important aspects of the simulation model will be discussed such that it becomes clear how it has been constructed. For making the simulating model, we rely on the results that have been obtained with process mining. However, as will become clear in this section, several of these results needed to be further analyzed in order that a simulation model is obtained that accurately mimics the various diagnostic and prosthetic steps in the AS-IS value chain. In other words, our aim is to build a simulation model that matches reality as close as possible for the current 'prosthesis' business process shown in Figure 2 and the current 'crown' business process shown in Figure 3. As a result, the predictions that will be derived from the simulation of the future value chain can therefore be believed to be reliable.

For obtaining these results, additional software algorithms needed to be made, which were not available in the ProM framework. Note that the discussion of the additional needed analyses holds for both the business process of placing a crown and the business process of placing a prosthesis. However, each analysis will only be illustrated in the context of the prosthesis business process.

\section{Performance Measures}

In order to measure the impacts of digital dentistry, clear performance indicators are needed which can be compared with each other. In general, regarding the application of digital dentistry, we expect reduced time efforts for patients, dentists, and dental labs. In particular, we distinguish the following performance indicators:

- Patients: the throughput time of the entire business process, i.e., the time that elapses from the start of the first task in the business process till the completion of the last task in the business process.

- Dentists: the time needed by the dentists to perform the tasks in the business process, i.e., the sum of the duration of the tasks that are performed by a dentist. 
- Dental lab technicians: the time needed by the dental lab technicians to perform the tasks in the business process, i.e., the sum of the duration of the tasks that are performed by a dental technician.

\section{Patients}

One of the most important artifacts in the model are the patients. In particular, for the discovery of the business process of placing a crown, data of 84 patients has been used which are both from the 'dental practice Wilhelminaweg' and 'ACTA'. Therefore, in the simulation model, 84 patients will follow the business process of placing a prosthesis (shown in Figure 2). This means that the execution of the required tasks in the process is simulated for each patient. In a similar fashion, 114 patients will follow the business process of placing a crown (shown in Figure 4).

\section{Execution of Tasks}

During simulation, tasks are performed by different kinds of resources. The way in which tasks are performed and the time spent on performing these tasks is configured in the following way:

- As we deal with data from 'ACTA', where the majority of the dentists are still in training, we have dentists which are experienced or inexperienced. This has an impact on the duration of tasks done by them. For example, for the 'intake' task, the average duration, when done by an experienced dentist, is 21.3 minutes (standard deviation: 12.8 minutes) whereas for an inexperienced dentist this is 56.3 minutes (standard deviation: 11.0 minutes). Furthermore, tasks done by dentists always correspond to an appointment. Therefore, for these tasks it has been decided that the duration follows a discrete probability distribution. For example, for the 'intake' task, 33\% of them took 30 minutes, and 33\% of them took 60 minutes in case of an experienced dentist. Next to that, the duration of such a task may also be determined by the number of implants that have been placed. For example, for the placing of one implant it takes on average 38.8 minutes (standard deviation: 23.1 minutes) by an experienced dentist whereas for two implants this is on average 64.6 minutes (standard deviation: 34.5 minutes) when done by an experienced dentist. Therefore, it has been decided that for tasks done after implantation and the implantation itself, the duration is determined by a discrete probability distribution which has the number of implants and the level of experience of the dentist as parameters. For tasks done before implantation, the level of experience of the dentist is the only parameter. Next to that, there are also tasks done by a lab technician. As we did not have data concerning the duration of these tasks, the distribution for these tasks has been determined based on interviews with lab technicians. Note that for these tasks also, we assume that once a lab 
technician has started working on a task, he will be working exclusively on that task till it is completed.

- As can be seen in Figure 3, patients may see different dentists. However, as also can be seen, patients may see the same dentist for multiple appointments after each other. So, before the start of each task, first, a probability is taken into account that the task is performed by the same dentist that did the previous task or not. If another dentist performs the task, we also have to take into account that the task is more likely to be done by an experienced dentist or the other way around. For example, if the 'fit prosthesis (front)' task needed to be done by another dentist then it was more often done by an inexperienced dentist (22\%) than an experienced dentist (3\%). Therefore, if a task is done by another dentist, then a second probability takes into account that there is a change from an inexperienced dentist to an inexperienced dentist, a change from an inexperienced dentist to an experienced dentist, a change from an experienced dentist to an experienced dentist, or a change from an experienced dentist to an inexperienced dentist.

\section{Time}

The majority of the tasks that are performed in the simulation model are performed by humans. Consequently, in the simulation model these tasks only need to be performed during office hours. However, there are also tasks that may be performed at any point in time. Therefore, in the model we adopt a 24 hour clock.

With regard to tasks that may only be performed during office hours (from 9 'o clock till 17 'o clock), this has been taken care of in the following way. Using ProM it was possible to obtain the average waiting time for each task. However, these figures where obtained without taking office hours into account. In case a distribution would be selected based on these figures, tasks may be started outside office hours. Therefore, in order to ensure that tasks only start during office hours and that time only elapsed within office hours, two steps needed to be taken. First, we implemented in ProM an algorithm such that it calculates the waiting time based on office hours. So, time which elapses outside office hours is not taken into account. Afterwards, the obtained waiting times for a task were fitted against a distribution. Second, based on the distribution, a value for the waiting time is sampled in the simulation model. This value determines how much time needs to elapse inside office hours before the task may start. Taking office hours into account it is calculated in the simulation model how much time (based on a 24 hour clock) needs to elapse before the task may start. For example, let's assume that the waiting time is 3 hours and that the current time is Friday at 16 'o clock. Now, the task may be started on Monday at 11 'o clock. 
With regard to the waiting times for tasks that may be started at any point in time, the obtained waiting times in ProM could directly be used to fit against a distribution. Also, the sampled waiting time could directly be used for determining when the task may be started based on a 24 clock.

The arrival process of patients for each of the two business processes also deserves some special attention. For both the 'dental practice Wilhelminaweg' and 'ACTA', it was only registered on which day the appointment was made for the intake. This means that for the arrival times of patients, the exact timestamp is not available even though in reality patients arrive throughout the day. As a consequence, patients arrive in groups. This makes that the stochastic process may not be modeled using a Poisson process which assumes that patients arrive one at a time. Therefore, we investigated whether the stochastic process can be modeled with a compound Poisson process which allows that patients arrive in batches. However, these batches still need to arrive on at a time and the interarrival times of batches need to follow an exponential distribution. For both business processes, a goodness-of-fit test (KolmogorovSmirnov test) showed that the interarrival times of batches could be modeled by an exponential distribution. Therefore, for both the business process of placing a crown $(\lambda=0.411)$ and the business process of placing a prosthesis $(\lambda=0.218)$, a compound Poisson process has been used. Note that for determining the arrival process only working days have been taken into account.

\section{Alternative Paths in a Process}

As can be seen in Figure 2 and Figure 4, there are multiple places in the process where alternative paths can be taken. As indicated earlier, there are tasks that are more likely to be done by an experienced dentist and there are tasks that are more likely to be done by inexperienced dentists. For example, at place ' $p 9$ ', the probability of taking the path to the 'fit prosthesis (front)' task is 0.03 for experienced dentists and 0.22 for inexperienced dentists. Therefore, in the simulation model, for the probability of taking an alternative path a difference is made in whether the previous task has been performed by an experienced or inexperienced dentist. In ProM, an algorithm needed to be implemented such that for the probability of selecting an alternative path, specific probabilities could be calculated for experienced and inexperienced dentists.

\section{Simulation Software}

As software for defining and executing the simulation model we have chosen CPN Tools. Consequently, the simulation model had to be defined in terms of a CPN model which can be executed in CPN Tools (7). CPNs provide a well-established and well-proven language suitable for describing the behavior of systems exhibiting characteristics such as concurrency, resource 
sharing, and synchronization. Furthermore, as a CPN is executable it can be used for performing simulation experiments. As a consequence, it can be used to evaluate the performance of different alternative designs.

\section{Validation}

The validation of a simulation model is a non-trivial but important step in the simulation process. By performing a validation it is determined whether the right model has been built (40). In (33) different approaches are mentioned that can be used for the validation of a simulation model. One of the most well-known approaches is to use historical data for validating the model. Moreover, as we are interested in the steady-state behavior of the system, it is important that aspects such as the warm up / cool down period, the length of a run and the number of replications are handled well. As the methods for realizing these aspects are allwell-established, the methods and outcomes for them are discussed briefly below. Finally, the validation of the model based on historical data is discussed.

\section{Warming up / cooling-down period, Run Length, and Number of Replications}

- Warming up / cooling-down period: For a steady state simulation model it is important that the steady-state behavior of the system is analyzed. As CPN Tools resets the model after each replication, the system always starts in an empty state. Moreover, as both business processes are simulated for a limited number of patients the system also ends in an empty state, i.e., when all cases have been handled. Therefore, as both the initial and the final state of the model do not represent a steady-state, a warm-up and coolingdown period must be considered (41). The warm-up period is the amount of time a model needs to come to a steady state whereas the cool-down period is the amount of time in which a model transitions from a steady-state to an empty state. One approach to determine the length of the warm-up period and the length of the cool-down period is to perform an estimation using time series (41).

For the 'prosthesis' business process it has been found to have the first 150 patients and the last 25 patients as warm-up and cool-down period respectively. For the 'crown' business process this appeared to be the first 215 and the last 55 patients respectively.

- Run Length: As a next step, it is necessary to determine the length of one single run. The length of the simulation runs must be long enough for the resulting data to be independent, i.e., the data values are not related to each other. One way to determine the run length is to choose a "reasonable" run length and then check whether the data is independent or not (33). One approach is to plot the data on a scatter diagram and visually inspect whether there is a dependency (33). 
For the both the 'prosthesis' and 'crown' it has been found graphically that the data is independent.

- Number of Replications: Finally, the number of replications needs to be determined. Replications are needed in order that results from different simulation experiments are independent and that they can be compared with each other using classical statistical procedures. In (33) a three-step method is provided which allows for calculating the number of replications based on a pre-specified precision of the collected data.

- As precision we have chosen for an error of $1.0 \%$ of the average value for both the 'prosthesis' and the 'crown' business process. In our opinion this is a reasonable error margin in order to reliably investigate the impacts of digital dentistry.

For this precision it appeared that 10 replications where needed for the 'prosthesis' business process and 19 for the 'crown' business process. However, the number of replications calculated by the approach may be seen as lowerbound. Therefore, in order to increase the reliability of the outcomes of the simulation experiments and that we had a powerful workstation at our disposal, it has been decided for both business processes to have 100 replications of the simulation model both for the validation of each business process and the subsequent experiments.

\section{Validation based on historical data}

As indicated before, the simulation model will be validated based on historical data. This comprises that output data of the simulation model closely resemble the output data that has been realized in reality. If the two sets of data 'closely' match, then the simulation model can be considered valid. For the performance measures described in the 'Performance Measures' Section, only for the performance indicator of the patient we had reliable historical data available. That is, for both business processes, using process mining, the average throughput time could be obtained. Of course, we would also have liked to validate the model using data for the other performance indicators, but no reliable historical data could be obtained for them.

In Table 2, the second column shows for both business processes the average throughput time that has been realized in reality. In the subsequent columns the results of the validation experiments have been given in which the simulation model has been configured as described above. For the simulation results, respectively, the average, standard deviation (SD), and the lower bound (LB) and upper bound (UB) for the corresponding 95\% confidence interval are shown. We see that for both business processes the average throughput time realized in reality is within the upper and lower bound of the confidence interval. Therefore, the simulation model is considered to be valid. 
Table 2: Validation. For a 95\% confidence interval, the average, standard deviation, lower bound, and upper bound values for each performance measure are presented. As performance measure the throughput time for the 'prosthesis' and the 'crown' business process are taken. All figures are presented in days.

\begin{tabular}{|l||c|c|c|c|c|}
\hline $\begin{array}{l}\text { Average } \\
\text { Throughput }\end{array}$ & Realization & \multicolumn{4}{|c|}{ Simulation (100 replications) } \\
\cline { 2 - 6 } $\begin{array}{l}\text { Time (ATP) } \\
\text { (in days) }\end{array}$ & Average & Average & $\begin{array}{c}\text { Standard } \\
\text { Deviation }\end{array}$ & $\begin{array}{c}\text { Lower Bound } \\
\text { (LB) }\end{array}$ & $\begin{array}{c}\text { Upper Bound } \\
\text { (UB) }\end{array}$ \\
\hline $\begin{array}{l}\text { 'Prosthesis' } \\
\text { process }\end{array}$ & 249.3 & 250.0 & 6.2 & 248.8 & 251.3 \\
\hline $\begin{array}{l}\text { 'Crown' } \\
\text { process }\end{array}$ & 232.0 & 231.1 & 5.4 & 230.1 & 232.3 \\
\hline
\end{tabular}

\section{Redesigned Business Processes}

In this section, several redesigns for investigating the impact of digital dentistry are described. In Table 1, it is shown which combination of techniques will be used for the 'prosthesis' and 'crown' business processes. These new techniques are the usage of an IOS for the making of the dental impression, the usage of computer guided surgery for the placement of the implants, and the usage of CAD/CAM for the production of the dental restoration.

For each redesigned business process, it will be discussed which steps are introduced and which steps are removed. For the new steps, it will also be indicated by which player the step is performed. With regard to timing information for the steps, it needs to be taken into account that for the combination of technologies that are introduced in the business process, currently no timing information is available for them together as they are currently not in use in the business process. In each case, it is discussed which comparable timing information has been selected. All together, the control-flow, resource, and performance perspectives of the TO-BE model in Figure 1 become clear.

\section{Redesign 1: Intra-Oral Scan of the Teeth and Digital Design of a Prosthesis}

As can be seen in Figure 2, for the production of the final prosthesis several steps need to be performed by both a dentist and the dental lab. This small subprocess starts with the making of the first impressions by the dentist (task " $D$ : first impressions" in Figure 2 ) and is completed when the prosthesis is finished in the dental lab (task "L:finish prosthesis" in Figure 2). Based on 
interviews with dental experts it is expected that within the coming five years it is not possible to digitize this entire subprocess. Nevertheless, as visualized in the redesigned business process in Figure 8, in order to start digitalize parts of the subprocess it is expected that the impression making can be replaced by an IOS. That is, from Figure 2, the tasks for the impression making (tasks "D:first impressions", "L:make individual impression trays", and "D:individual impressions") are replaced by respectively the making of an IOS (task "D:make Intra-Oral Scan" in Figure 8), the processing of the scan (task "S:process scan" in Figure 8), and the production of an SLA model of the patient's teeth (task "S:sinter SLA model" in Figure 8). Also, it is expected that for the last step of the dental lab in which the prosthesis is finalized, the bar for the final prosthesis can be produced using CAD/CAM techniques. That is, the "L:finish prosthesis" task of Figure 8 can be divided into several tasks in which first a scan is made of the wax model that has been used for the fitting of the prosthesis (task "L:scan wax model" in Figure 2). The scan of this model, together with the processed scan of the IOS (task "L:import Intra-Oral Scan" in Figure 8), is used for the subsequent design (task "L:design bar" in Figure 8) and sintering of the bar (task "S:sinter bar" in Figure 8). Finally, after the bar has arrived in the $l a b$, the prosthesis can be finished.

As can be seen in Figure 8, one new role is introduced. The prefix " $\mathrm{S}$ " indicates that a person in a milling center is needed for doing a task. 


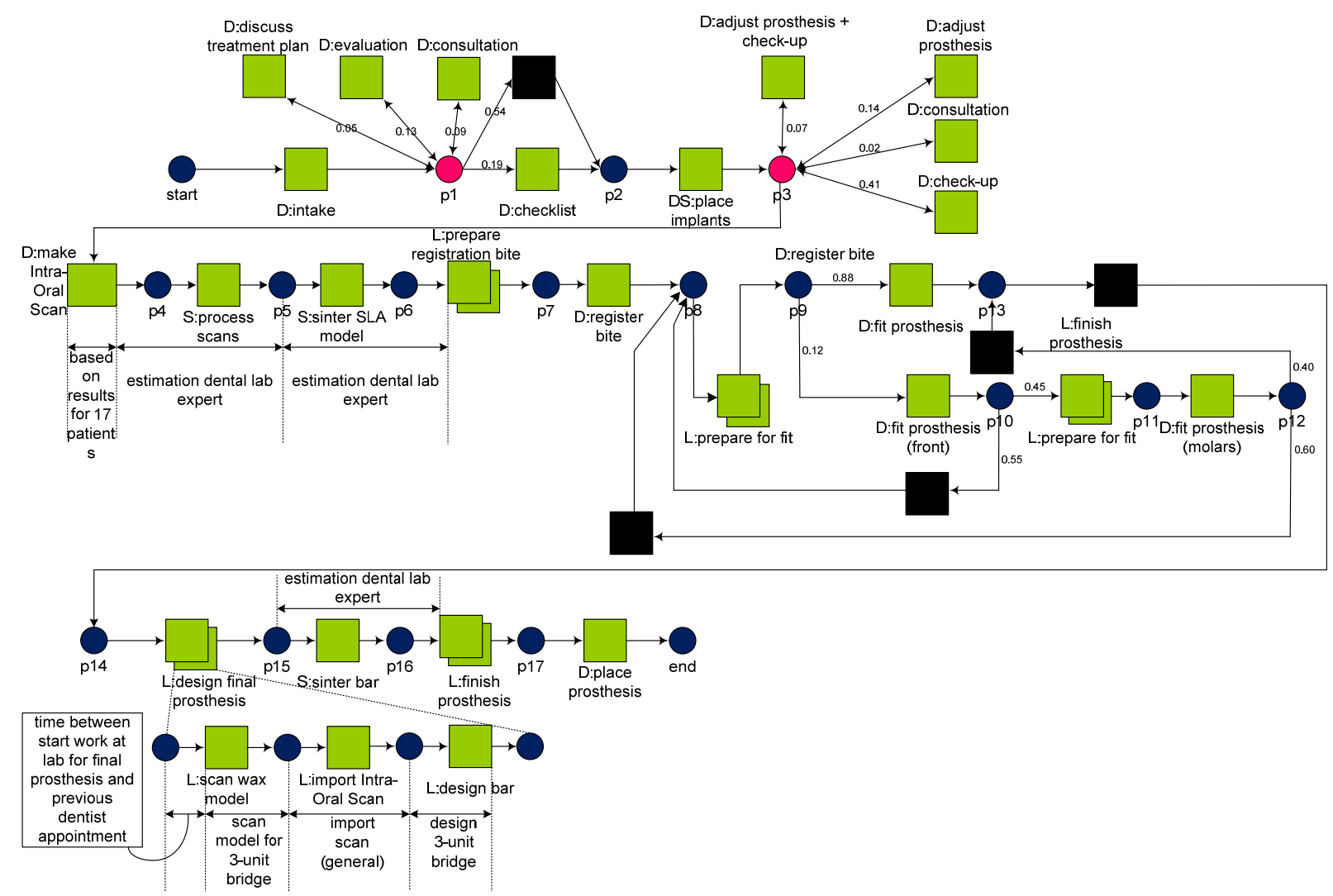

Figure 8: Redesigned 'prosthesis' business process. Instead of an impression using an impression tray an IOS of the teeth is made and the final prosthesis is made using CAD/CAM techniques.

The timing information of the new tasks are based on existing data or this has been estimated based on the experiences of experts. For each task, this is as follows:

- D:make IOS: the task is performed immediately following the previous task. Therefore, there is no waiting time. The execution time is based on existing data that involves digital impressions that have been made for 17 patients (see Section "Intra-Oral Scanning").

- S: process scans: No execution data was available for this task. Therefore, the time that elapses from the end of the intra-oral scanning till the time the processed scan arrives in the lab was based on interviews with several lab experts. Their estimation depended on comparable experiences in which an IOS was made for patients without implants and that afterwards the processed scan needs to be send to the lab. Note that after the processed scan has arrived in the lab, the lab needs to trigger the production of the SLA model.

- S: sinter SLA model: Also no execution data was available for this task. Therefore, the time that elapses from the end of the processing of the scan till the time the SLA model arrives in the lab was based on interviews with several lab experts. Their estimation depended on 
comparable experiences in which an SLA model was produced for patients without implants.

- L: design bar: Regarding the design of a bar, no execution data from the Dentalcam dental lab was available (see Section "Digital Design of Crowns and Bridges"). However, based on discussions with dental lab experts, it was decided that the design of a 3-unit bridge is a representative alternative. Therefore, for the scanning of the wax model and the design of the bar, timing information from the scanning of a wax model for a 3-unit bridge and from the design of a 3-unit bridge has been used respectively. Also, for the import of an IOS no execution data was available. As an alternative, timing information for the import of an existing scan has been taken. Furthermore, after the fitting of the prosthesis by the dentist, typically some time elapses before the lab starts working on designing a bar. As an alternative for this waiting time, the waiting time has been taken that exists for starting the work in the lab for finishing the prosthesis in the AS-IS situation, i.e. the waiting time that exists for the "L:finish prosthesis" task in Figure 2.

For the simulation experiment, the business process as visualized in Figure 8 , has been simulated. Remember, that for the dental lab it was only recorded on which day the activities have been executed and that therefore not very precise information has been obtained about the waiting time for the activities done by the dental lab. Moreover, it is also not clear whether a small change in the waiting time leads to a major change in the throughput time of the entire process. In order to test for the robustness of the throughput time of the entire process based on the waiting time for the lab, 4 additional variants have been simulated in which the waiting time for the dental lab activities is both increased and decreased by $10 \%$ and $20 \%$. 


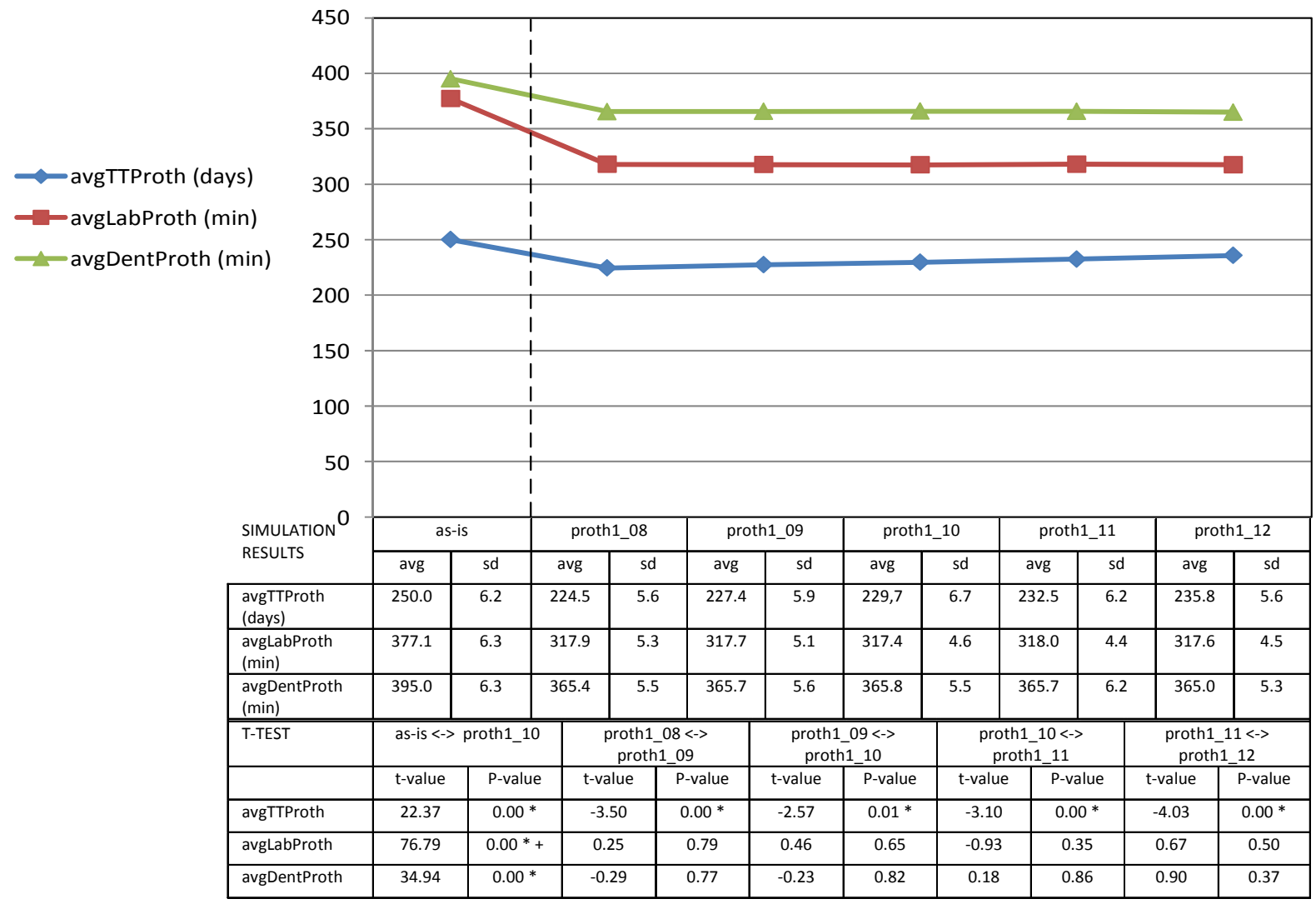

Figure 9: Results for the experiments in which an IOS of the teeth is made and the final prosthesis is made using CAD/CAM techniques. For the total throughput time of the entire business process (avgTTProth), the average total time spent by people in the lab (avgLabProth), and the average total time needed by the dentist to perform the tasks in the business process (avgDentProth), the average (avg) and standard deviation (sd) of 100 runs are shown in the 'simulation results' table part. In addition, in the 'T-TEST' table part, the result of $t$-tests are shown to determine whether the observed average of two experiments is statistically significant from zero. For each simulated situation, the average for each performance measure is visualized in the graph.

For the business process and each of its four variants, 100 replications of the simulation model have been carried out to be able to determine standard deviations. The obtained results can be seen in Figure 9 which focuses on the three performance indicators that have been defined in order to measure the impact of digital dentistry on a certain business process. Figure 9 is splitup in three parts. First, for the business process and each of its variants, the average for each performance indicator is visualized in the graph. Second, the table named "SIMULATION RESULTS" shows first for every performance indicator the average waiting time (avg) and 
standard deviation that has been obtained for the validated model. For the redesigned model, the next rows show for each performance indicator the figures that are obtained in case the waiting time for the lab activities is decreased by $20 \%$ (proth1_08), is decreased by $10 \%$ (proth1_09), is unmodified (proth1_10), is increased by 10\% (proth1_11), and is increased by 20\% (proth1_12).

The table with name "T-TEST" shows the result of the t-tests in order to determine whether the observed average for a certain performance indicator is statistically significant from zero between two simulated situations. Respectively, the outcome of t-tests for the validated model and the situation in which the waiting times for the lab activities is unmodified are added ('ASIS <-> proth1_10); the situation in which the waiting time for the lab activities is decreased by $20 \%$ and the situation in which the waiting time for the lab activities is decreased by $10 \%$ are added ('proth1_08<-> proth1_09'); the situation in which the waiting time for the lab activities is decreased by $10 \%$ and the situation in which the waiting time for the lab activities is unmodified are added ('proth1_09 <-> proth1_10'); the situation in which the waiting time for the lab activities is unmodified and the situation in which the waiting time for the lab activities is increased by $10 \%$ are added ('proth1_10 <-> proth1_11'); and the situation in which the waiting time for the lab activities is increased by $10 \%$ and the situation in which the waiting time for the lab activities is increased by $20 \%$ are added ('proth1_11 <-> proth1_12') are shown. More specifically, the t-test has been constructed to determine whether the difference between the average of two situations equals 0.0 (null hypothesis) versus the alternative hypothesis that the difference does not equal 0.0 at the $95 \%$ confidence level $(\alpha=0.05)$. It is assumed that the observed values for each situation come from a normal distribution and that the variances of them are not equal. In case $P<0.05$ (in the column 'P-value'), this implies that the null hypothesis is rejected. For example, the difference of the average total throughput time of the entire business process for the validated model ('AS-IS') and the situation of the redesigned business process in which the waiting time for the lab activities is unmodified ('proth1_10') is statistically significant because $P=0.00$.

When comparing the results for the validated model and the redesigned model in which the waiting times for the lab activities are unmodified, it can be seen that for the redesigned model the average total throughput time of the business process is approximately 20 days lower than for the validated model and is significant $(P=0.00)$. For the average total time spent by people in the lab, this difference is around 60 minutes lower (statistically significant, $P=0.00$ ) whereas for the average total time spent by a dentist, this difference is around 29 minutes lower (statistically significant, $P=0.00$ ). In case the waiting time for the lab activities in the redesigned business process is modified by $10 \%$ or $20 \%$, it can be seen that this leads to significantly 
different results for the average total throughput time of the entire business process. However, for the situation in which the waiting time is reduced by $20 \%$ and the situation in which the waiting time is increased by $20 \%$, the difference is 11.3 days. This shows that the average total throughput time of the entire business process is not dramatically impacted.

In general, it can be seen that for the redesigned business process there is quite some impact for people in the lab as less work needs to be performed by them. Also, for patients, there is quite some impact on the total treatment time as on average one appointment less is needed for the impression making and which saves the waiting time for the appointment. For the dentist, there is the least benefit with regard to the total working that needs to be done as instead of two times making an impression using an impression tray and impression is made using an IOS.

\section{Redesign 2: Mini Implants, Computer Guided Surgery, and Digital Design of a} Prosthesis

As also indicated for the previous redesign, within five years it is not possible to digitize the entire process of the production of the prosthesis. However, for digitizing parts of the 'prosthesis' business process a complete different approach is possible in which in the beginning of the treatment process 3-mini implants are placed. Furthermore, in the end of the treatment process the final prosthesis is designed using CAD/CAM, a drilling guide is made, and finally, the implants are placed using the drilling guide and the final prosthesis is immediately placed. The redesigned business process can be seen in Figure 10.

When comparing Figure 10 with Figure 2, it can be seen that there are some major changes in comparison to the AS-IS situation. In the beginning, 3 mini-implants are placed (task "DS:place 3 mini-implants" in Figure 10) instead of regular implants (task "DS:place implants" in Figure 2). During the appointment for the placing of the 3-mini implants immediately an individual impression is made such that the lab can work on preparing the registration of the bite (task "L:prepare registration bite" in Figure 2). So, an individual impression (task "D:first impressions" in Figure 2) and the making of an individual impression tray (task "L:make individual impression trays" in Figure 2) is not needed anymore. Also, the tasks typically done between the placing of the implants and the making of the first impressions are not needed anymore (tasks "D:adjust prosthesis", "D:adjust prosthesis + check-up", and "D:consultation”, “D:check-up" in Figure 2). Later in the business process, the final prosthesis of the patient is made using CAD/CAM techniques. However, the prosthesis of the patient contains barium elements such that before finalizing the prosthesis a CT-scan is made of the patient having the fitted prosthesis in the mouth (task "D:make CT-scan" in Figure 10). Afterwards, the CT-scan is used for the 
preoperative planning such that the drilling guide can be made and that the bar and structure of the final prosthesis can be designed (task "L:preoperative planning and design final prosthesis" in Figure 10). Once the bar and structure of the final prosthesis are sintered and the drilling guide is milled (task "S:sinter bar and structure + mill drilling guide" in Figure 10), the prosthesis can be finished in the lab (task "L:finish prosthesis" in Figure 10). Finally, the implants and the prosthesis are placed in one appointment (task "D:place implants and prosthesis" in Figure 10).

As can be seen in Figure 10, one new role is introduced. The prefix " ${ }^{T}$ " indicates that a radiology assistant is needed for making a CT-scan. 


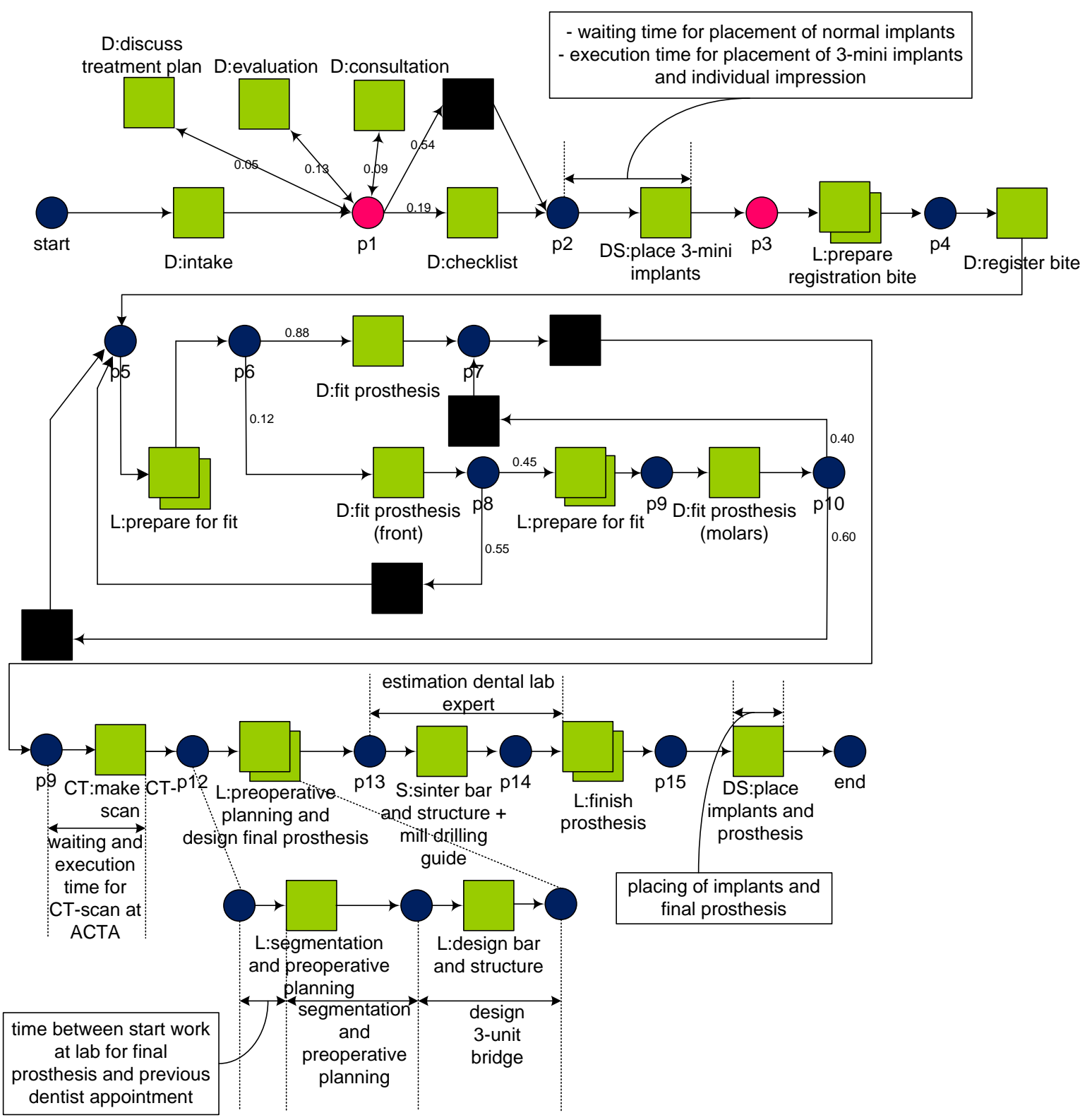

Figure 10: Redesigned 'prosthesis' business process. In the business process three mini implants are placed in the beginning. In the end, the final implants are placed using guided surgery, and the final prosthesis is made using CAD/CAM techniques.

Also here, the timing information of the new tasks is based on existing data or this has been estimated based on the experiences of experts. As the derivation of this information proceeded in a similar fashion as for the first redesign, this aspect is not elaborated in further detail but is therefore only illustrated graphically in Figure 10. 
For the simulation experiment, the business process as visualized in Figure 10 has been simulated. Similarly as for the previous redesign, in order to test for the robustness of the throughput time of the entire process based on the waiting time for the lab, 4 additional variants have been simulated in which the waiting time for the dental lab activities is both increased and decreased by $10 \%$ and $20 \%$.

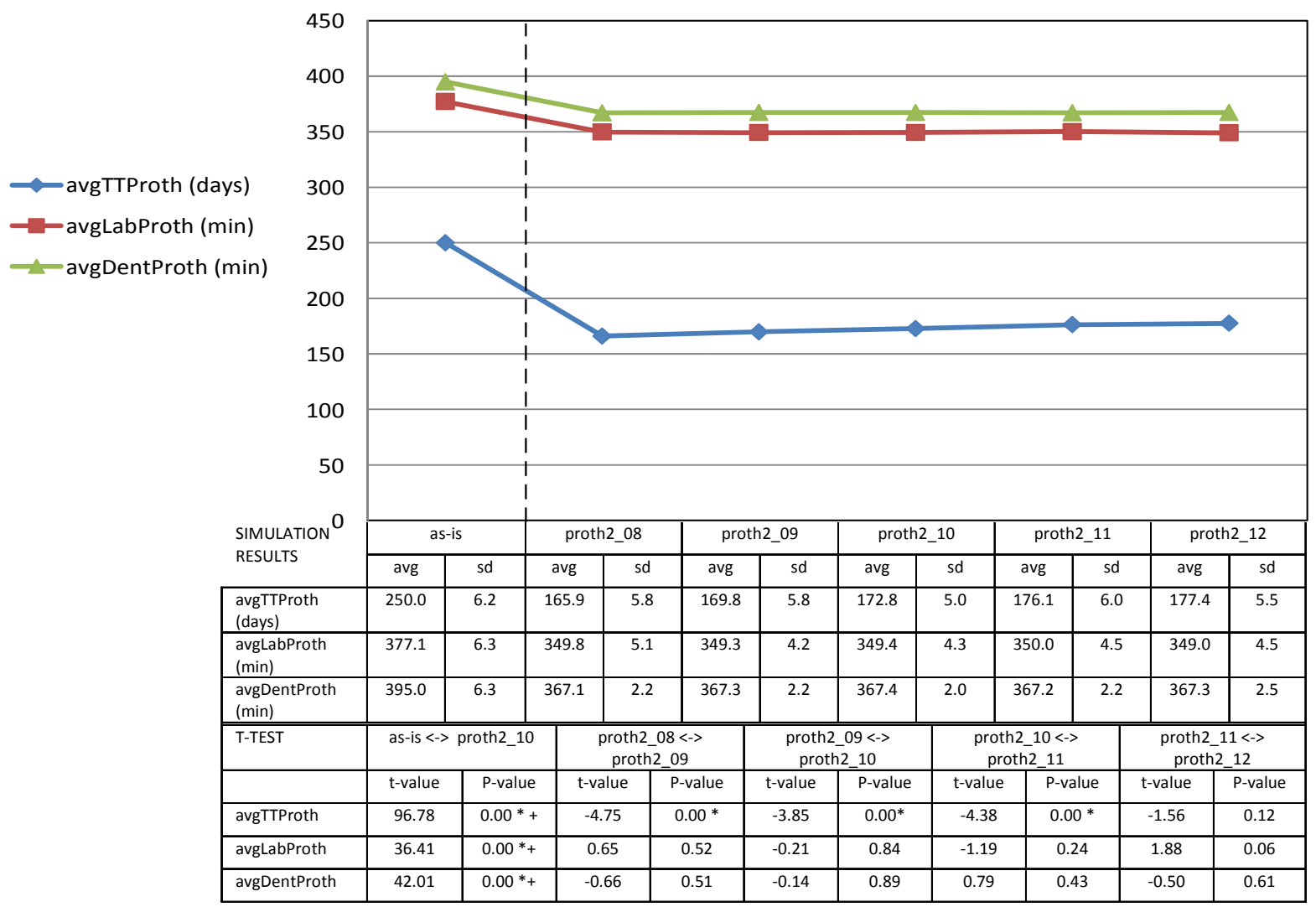

Figure 11: Results for the experiments in which three mini implants are placed, the implants are placed using guided surgery, and the final prosthesis is made using CAD/CAM techniques. For the total throughput time of the entire business process (avgTTProth), the average total time spent by people in the lab (avgLabProth), and the average total time needed by the dentist to perform the tasks in the business process (avgDentProth), the average (avg) and standard deviation (sd) of 100 runs are shown in the 'simulation results' table part. In addition, in the ' $T$ TEST' table part, the result of t-tests are shown to determine whether the observed average of two experiments is statistically significant from zero. For each simulated situation, the average for each performance measure is visualized in the graph. 
For the business process and each of its four variants, 100 replications of the simulation model have been carried out to be able to provide standard deviations. The obtained results can be seen in Figure 11 which focuses on the three performance indicators that have been defined in order to measure the impact of digital dentistry on a certain business process. Figure 11 . Note that the graph and the two tables ("SIMULATION RESULTS" and "T-TEST") have been set-up in a similar fashion as for the first redesign.

When comparing the results for the validated AS-IS model and the redesigned TO-BE model in which the waiting times for the lab activities are unmodified, it can be seen that for the redesigned model the average total throughput time of the business process is around 77 days lower than for the validated model and is significant $(P==0.00)$. For the average total time spent by people in the lab, this difference is around 28 minutes lower (statistically significant, $\mathrm{P}$ $=0.00$ ) whereas for the average total time spent by a dentist, this difference is around 29 minutes lower (statistically significant, $P=0.00$ ). In case the waiting time for the lab activities in the redesigned business process is modified by $10 \%$ or $20 \%$, it can be seen that this leads to significantly different results for the average total throughput time of the entire business process. However, this difference is at most 11.5 days which means that the average total throughput time of the entire business process is softly impacted.

In general, it can be seen that for the redesigned business process there is a comparable impact for both the dentist and the people in the lab. For the dentist, some less work is needed and for the people in the lab also some less work is needed. For patients there is a major benefit. That is, also taking into account the results for the previous redesign for the prosthesis, it can be seen that there is a large reduction on the total treatment time. This is mainly due to the fact that the healing time after the placing of the implants is not included in the business process anymore. Also, one appointment less is needed for the making of an impression.

\section{Redesign 3: Intra-Oral Scan of the Teeth and Digital Design of a Crown}

For the production of a crown it is currently required to make two impressions. Also, the crown needs to be produced using wax. During this process, only analogue artifacts are used which are all subject to loss of precision. These inaccuracies may lead to rework and less patient comfort due to a non-precisely fitting crown. Therefore, in Figure 12, a business process is presented in which the production of the final crown is primarily done using digital artifacts thereby avoiding the loss-of-precision problem. Instead of an individual impression and the subsequent production of a plaster model (tasks "L:make individual impression trays" and "D:make impressions" in Figure 4), an IOS of the mouth is made (task "D:make Intra-Oral-Scan" in Figure 12). After the processing of the scan in a milling center (task "S:process scans" in Figure 12), it is 
used by the dental lab for designing the abutment and coping of the crown (task "L:design crown" in Figure 12). The coping and abutment are milled in a milling center (task "S:mill coping and abutment" in Figure 12) after which it can be finalized in the lab (task "L:finish crown" in Figure 12) and sent to the dentist. The design and milling of the structure of the crown replaces the production of the crown using wax (task "L:make crown" in Figure 4).

As can be seen in Figure 12, one new role is introduced. The prefix " $\mathrm{S}$ " indicates that a person in a milling center is needed for performing a task.

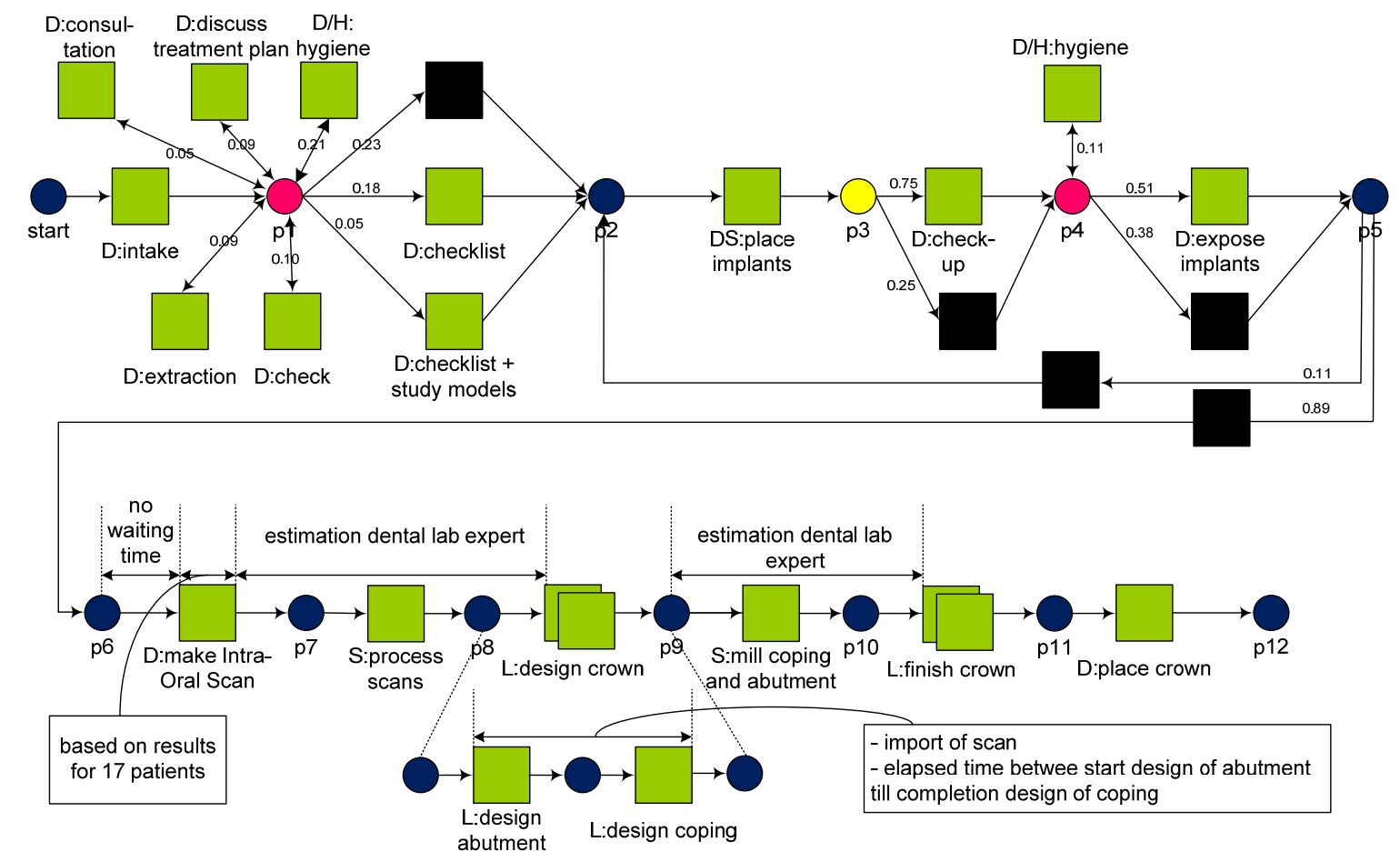

Figure 12: Redesigned 'crown' business process. Instead of an impression using an impression tray an IOS of the teeth is made and the final crown is made using CAD/CAM techniques.

Also here, the timing information of the new tasks is based on existing data or this has been estimated based on the experiences of experts. As the derivation of this information proceeded in a similar fashion as for the first redesign, this aspect is not elaborated in further detail but is therefore only illustrated graphically in Figure 12. 
For the simulation experiment, the business process as visualized in Figure 12, will be simulated. Similarly as for the two previous redesigns, 4 additional variants have been simulated in which the waiting time for the dental lab activities is both increased and decreased by $10 \%$ and $20 \%$.

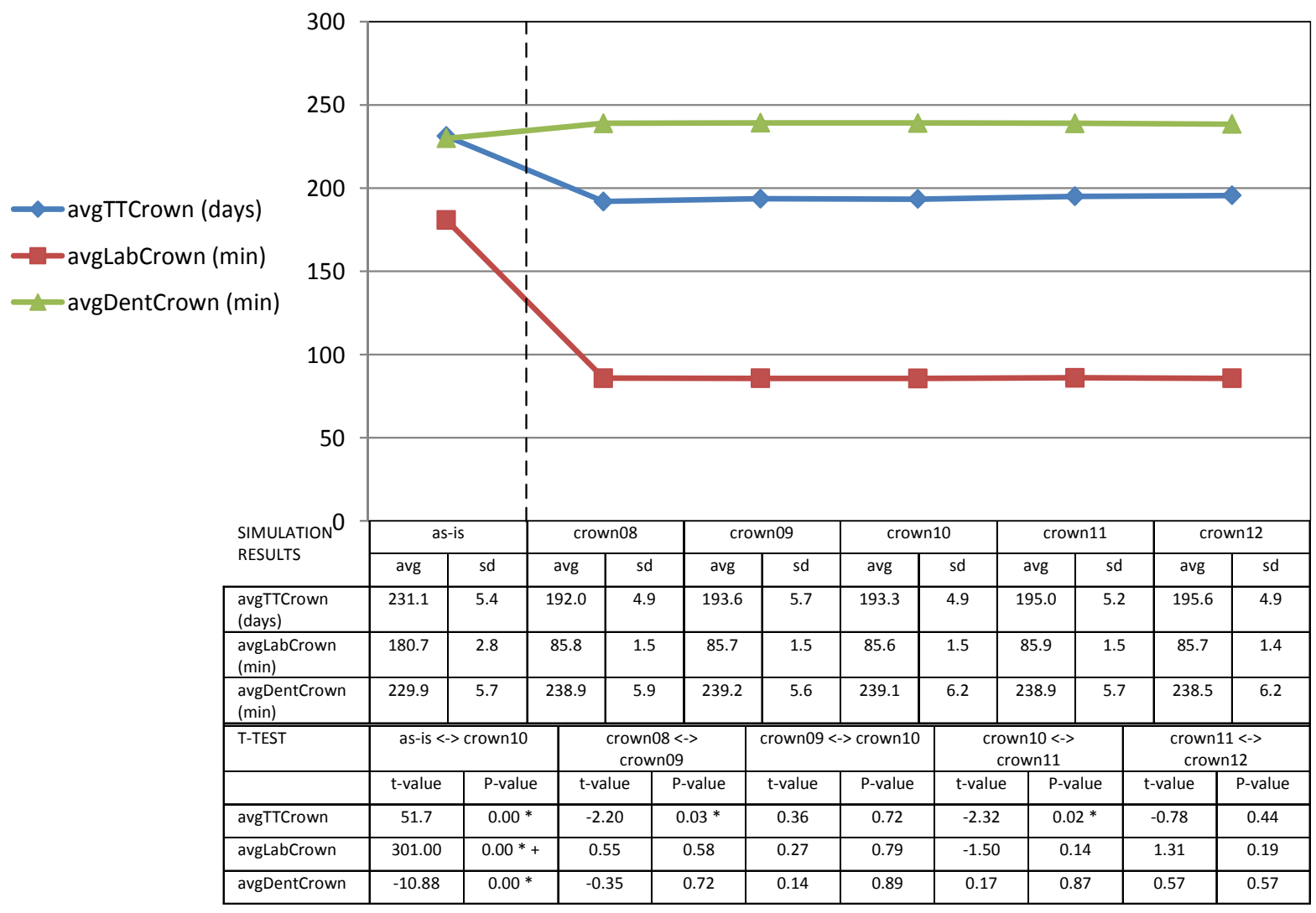

Figure 13: Results for the experiments in which an IOS of the teeth is made and the final crown is made using CAD/CAM techniques. For the total throughput time of the entire business process (avgTTCrown), the average total time spent by people in the lab (avgLabCrown), and the average total time needed by the dentist to perform the tasks in the business process (avgDentCrown), the average (avg) and standard deviation (sd) of 100 runs are shown in the 'simulation results' table part. In addition, in the 'T-TEST' table part, the result of t-tests are shown to determine whether the observed average of two experiments is statistically significant from zero. For each simulated situation, the average for each performance measure is visualized in the graph.

For the business process and each of its 4 variants, 100 replications of the simulation model have been carried out to be able to provide standard deviations. The obtained results can be 
seen in Figure 13 which focuses on the three performance indicators that have been defined in order to measure the impact of digital dentistry on a certain business process. Note that also here the graph and the two tables ("SIMULATION RESULTS" and "T-TEST") have been set-up in a similar fashion as for the first redesign.

When comparing the results for the validated model and the redesigned model in which the waiting times for the lab activities are unmodified, it can be seen that for the redesigned model the average total throughput time of the business process is around 38 days lower than for the validated model and is significant $(P=0.00)$. For the average total time spent by people in the $l a b$, this difference is around 95 minutes lower (statistically significant, $P=0.00$ ) whereas for the average total time spent by a dentist, this difference is around 9 minutes higher (statistically significant, $P=0.00$ ). In case the waiting time for the lab activities in the redesigned business process is modified by $10 \%$ or $20 \%$, it can be seen that this leads to significantly different results for the average total throughput time of the entire business process. However, this difference is at most 1.7 days which means that the average total throughput time of the entire business process is hardly impacted.

In general, it can be seen that for the redesigned business process there is quite some impact for people in the lab as a substantial amount of work is not needed anymore. Also, for patients, there is quite some impact on the total treatment time as on average one appointment less is needed for the impression making. For the dentist, there is even a small drawback as on average making an impression using an IOS costs a bit more time than making an impression using an impression tray.

\section{Discussion}

As indicated in the introduction, this paper served two purposes. From a scientific point of view, a process-oriented methodology has been proposed for evaluating the impact of IT on a process which consists of multiple distributed actors. In this method, both process mining and discrete event simulation are key ingredients. From a practical point of view and to illustrate the process-oriented methodology, a concrete case has been discussed in which the effects of digital dentistry on the implant value chain have been investigated using process mining and discrete event simulation. In this section, we first reflect on the outcomes of the simulation experiments that have been performed for the three redesigned business processes in order to investigate the impact of digital dentistry on the process-oriented methodology for evaluating 
the impact of IT. Second, we reflect on the process-oriented methodology for evaluating the impact of IT.

\section{Case: the Impact of Digital Dentistry}

Below, the outcomes of the simulation experiments for the three redesigned business processes will be discussed.

First of all, for the two redesigned 'prosthesis' business processes and the redesigned 'crown' business process, it becomes clear that both for the lab and the patient there are major benefits. In the lab, less work is needed due to the introduction of an Intra-Oral Scanner, guided surgery, and the design of a crown and prosthesis using CAD/CAM techniques. For the patient, the total treatment is considerably reduced. Surprisingly, for the dentist, the introduction of the aforementioned technologies leads hardly to any benefits in the sense that the working time that is needed for a patient is hardly reduced or even decreases. So, only slightly more patients can be treated by a dentist within the same time. Even more, a dentist themself is responsible for the purchasing of an Intra-Oral Scanner which is an expensive device (suggested retail price is $\left.€ 25,000^{\prime}\right)$. Given this reasoning, instead of being a nice 'gadget', it will be hard to convince dentists to purchase IOS. A similar reasoning can be made regarding the introduction of a CTscanner in a dental clinic or the usage of software for guided surgery. Rather, the dental lab, which has large benefits by the introduction of digital technologies, seems the more appropriate party to introduce new technologies in the practice of a dentist. Such a development would require major changes in the mindset of both dental lab owners and dentists. Also, the above is a different insight than expected.

Second, from a patient perspective, the simulation experiments made clear that the introduction of an IOS has as effect that on average one visit less to the dentist is needed. During the discussion of the current business processes for the 'crown' and 'prosthesis' it became clear that a major part of the patient treatment time is consumed by the time in which the bone is healed around the placed implants. For the second redesign, a design has been presented for the 'prosthesis' business process in which for the treatment period it was possible to eliminate the time that is needed for the healing. This significantly reduced the total throughput time as in the last appointment both the implants and the final prosthesis are placed. In that sense, for the placement of crown business process it can be anticipated that a similar approach also leads to a main reduction of the average total treatment time. As on average the healing time is 3 months after the placing of crown, this would lead to a comparable reduction in the average total treatment time. However, a medical drawback is 
that complications might occur because of the immediate load on the implant due to the placed crown.

\section{Process-oriented Methodology for Evaluating the Impact of IT}

When focusing on the above obtained insights for the dental case it may be concluded that these are not trivial. This illustrates the value of a process-oriented analysis in which the impacts of IT are investigated. We have proposed an approach in which both process mining and discrete event simulation are used. As process mining uses factual execution data it allows for obtaining an objective view on how processes are really executed. People that are involved in the processes under study typically only have a limited or idealized view on how these processes are executed. That is, they tend to have an ideal scenario in mind, which in reality is only one of the many scenarios possible. Additionally, using process mining, quantitative insights concerning the processes under study can be obtained. Via discrete event simulation, the impact of different digital technologies can be analyzed and compared. For each introduced technology, several variants to it can be easily investigated. Additionally, as the simulation model is created using process mining, the actual simulation phase can be started much quicker compared to the traditional approach, where simulation models are created manually.

For the dental case, we have focused on three different perspectives, i.e. the control-flow, resource and performance perspective, and on three different performance indicators, i.e. the total throughput time, the total time spent by people in the lab, and the total time spent by a dentist. However, our methodology is generic such that other perspectives, e.g. the data perspective, and other performance indicators can easily be included, if needed. Additionally, there are no constraints regarding the process mining algorithms that can be applied. However, dependent on the goal of the simulation model, a limitation may be that no process mining algorithm may exist in order to obtain the desired information. Currently, many researchers are working on the topic and new algorithms are continuously developed.

Till now, the proposed process-oriented methodology has only been applied for one extensive case. However, we believe that the approach can easily be applied to other cases and in other domains.

\section{Conclusion}

In this paper, we have proposed a process-oriented methodology for investigating the impacts of IT. The methodology is based on both process mining and discrete event simulation. By using process mining, an objective view is obtained on how processes are really executed. 
Furthermore, the actual simulation phase in which impacts of digital technologies are evaluated can be started much quicker compared to the traditional approach, where simulation models are created manually. Also, the methodology allows for obtaining non-trivial process insights that by following another methodology perhaps would not have been obtained.

As future work we plan to extend the work of Rozinat et al. [REF] in order that results obtained for various perspectives (e.g. the control, resource, and data perspective) can be (semi-) automatically stitched together in one comprehensive simulation model. Closely, related to this is the extension of the framework with additional perspectives such as cost and precision (e.g. precision of the placed implants or precision of the produced dental restoration).

Also, in order to evaluate the general applicability of our approach, we plan to apply our approach in other domains (e.g. the healthcare and the automotive domain).

\section{Acknowledgement}

This project was supported by a grant from the ITI foundation for the Promotion of Oral Implantology, Switzerland. 


\section{References}

[1] Devaraj, S. and Kohli, R.. Performance Impacts of Information Technology: Is Actual Usage the Missing Link. Management Science 49 (3), 273-289. 2003.

[2] Venkatesh, V., Morris, M. G., Davis, G. B., and Davis, F. D.. User Acceptance of Information Technology: Toward a Unified View. MIS Quarterly 27 (3), 425-478. 2003.

[3] Seddon, P., Staples, S., Patnayakuni, R., and Bowtell, M.. Dimensions of Information Systems Success. Communications of AIS 2. 1999.

[4] Grover, V., Jeon, S. R., and Segars, A. H.. Information Systems Effectiveness: The Construct Space and Patterns of Application. Information and Management 31, 177-191. 1996.

[5] DeLone, W. H. and McLean, E. R.. The Delone and McLean Model of Information Systems Success: A Ten-Year Update. Journal of Management Information Systems 19(4), 9-30. 2003.

[6] van der Aalst, W. M. P.. Process Mining: Discovery, Conformance and Enhancement of Business Processes. 2011. Springer-Verlag.

[7] Jensen, K., Kristensen, L. M., and Wells, L.. Coloured Petri Nets and CPN Tools for Modelling and Validation of Concurrent Systems. International Journal on Software Tools for Technology Transfer 9(3-4), 213-254. 2007.

[8] Benalouane, A., Bakker, Q., Wismeijer, D., and Genuchten, M. v.. Digital Dentistry, Promise, reality and the role of software standards. Digital Dental News . 2011.

[9] Shannon, R. E.. Systems Simulation: the Art and Science. 1975. Prentice-Hall, Englewood Cliffs.

[10] Dahl, O. J. and Nygaard, K.. SIMULA: An ALGOL Based Simulation Language. Communications of the ACM 1, 671-678. 1966.

[11] Kelton, D. W. and Sturrock, D.. Simulation with Arena. 2003. McGraw-Hill, New York.

[12] Hall, C. and Harmon, P.. A Detailed Analysis of Enterprise Architecture, Process Modeling, and Simulation Tools. BPTrends, Technical report 2.0 . 2006.

[13] Jansen-Vullers, M. and Netjes, M.. Business Process Simulation - A Tool Survey. Jensen, K. Workshop and Tutorial on Practical Use of Coloured Petri Nets and the CPN Tools . 2012.

[14] FileNET. FileNET.

[15] Perceptive Software. BPM|One.

[16] Bizflow. Bizflow.

[17] IBM. IBM WebSphere.

[18] Jun, J. B., Jacobson, S. H., and Swisher, J. R.. Application of Discrete-Event Simulation in Healthcare Clinics: A Survey. Journal of the Operational Research Society 50, 109123. 
[19] England, W. and Roberts, S.. Applications of Computer Simulation in Health Care. Highland, H. J., Hull, L. G., and Neilsen, N. R. Proceedings of the 1978 Winter Simulation Conference , 665-676. 1978. Institute of Electrical and Electronics Engineers.

[20] Yang, Y., Sullivan, K., Wang, P., and Naidu, K. D.. Applications of Computer Simulation in Medical Scheduling. Proceedings of the Joint Conference on Information Sciences . 2000.

[21] Rozinat, A., Mans, R. S., Song, M., and van der Aalst, W. M. P.. Discovering Simulation Models. Information Systems 34, 305-327. 1-1-2009. Elsevier.

[22] Bozkaya, M., Gabriels, J., and van der Werf, J.. Process diagnostics: a method based on process mining. International Conference on Information, Process, and Knowledge Management (eKNOW' '09) (2009) , 22-27. 2009.

[23] Rebuge, A. and Ferreira, D. R.. Business process analysis in healthcare environments: A methodology based on process mining. Information Systems 37, 99-116. 2011.

[24] Zhou, W. and Piramuthu, S.. Framework, Strategy and Evaluation of Health Care Processes with RFID. Decision Support Systems 50, 222-233. 2010.

[25] Marthandan, G. and Tang, C. M.. Information Systems Evaluation: An Ongoing Measure. International Journal of Business Information Systems 6 (3), 336-353. 2010.

[26] Thomas, G. and Fernandez, W.. Success in IT Projects: a Matter of Definition? International Journal of Project Management 26 (7), 733-742. 2008.

[27] Frisk, E.. Categorization and Overview of IT Evaluation Perspectives - A Literature Review. Remenyi, D. Proceedings of the European Conference on Information Management and Evaluation , 159-170. 2007.

[28] Cronk, M. C. and Fitzgerald, E. P.. Understanding IS Business Value: Derivation of Dimensions. Logistics Information Management 12, 40-49. 1999.

[29] Smithson, S. and Hirschheim, R.. Analysing Information Systems Evaluation: Another Look at an Old Problem. European Journal of Information Systems 7, 158-174. 1998.

[30] Ammenwerth, E., Brender, J., Nykänen, P., Prokosh, H.-U., Rigby, M., and Talmon, J.. Visions and Strategies to Improve Evaluation of Health Information Systems: Reflections and Lessons Learned Based on the HIS-EVAL Workshop in Innsbruck. International Journal of Medical Informatics 73, 479-491. 2004.

[31] Yusof, M. M., Papazafeiropoulou, A., Paul, R. J., and Stergioulas, L. K.. Investigating Evaluation Frameworks for Health Information Systems. International Journal of Medical Informatics 77, 377-385. 2008.

[32] van der Aalst, W. M. P., Reijers, H. A., Weijters, A. J. M. M., van Dongen, B. F., de Medeiros, AK. A. d., Song, M., and Verbeek, H. M. W.. Business Process Mining: An Industrial Application. Information Systems 32(5), 713-732. 2007.

[33] Law, A. M.. Simulation Modeling \& Analysis (4th Edition). 2007. McGraw-Hill. 
[34] American Dental Association. Dentistry Definitions (http://www.ada.org/495.aspx). 2012.

[35] van der Aalst, W. M. P. and van Hee, K. M.. Workflow Management: Models, Methods, and Systems. 2002. The MIT Press.

[36] Song, M. and van der Aalst, W. M. P.. Supporting Process Mining by Showing Events at a Glance. Chari, K. and Kumar, A. Proceedings of theSeventeenth Annual Workshop on InformationTechnologies and Systems (WITS 2007), 139-145. 2007.

[37] Kapos, T., Ashy, L. M., Gallucci, G. O., Weber, H. P., and Wismeijer, D.. Computeraided Design and Computer-assisted Manufacturing in Prosthetic Implant Dentistry. The International Journal of Oral \& Maxillofacial Implants 24 (Suppl: 110-7). 2009.

[38] van der Meer, W. J., Andriessen, F. S., Wismeijer, D., and Ren, Y.. Application of IntraOral Dental Scanners in the Digital Workflow of Implantology. PLoS ONE 7(8). 2012.

[39] Jung, R. E., Schneider, D., Ganeles, J., Wismeijer, D., Zwahlen, M., Hämmerle, C. H. F., and Tahmaseb, A.. Computer Technology Applications in Surgical Implant Dentistry: A Systematic Review. The International Journal of Oral \& Maxillofacial Implants 24 (Suppl: 92-109). 2009.

[40] Banks, J., Carson, J. S., and Nelson, B. L.. Discrete-Event System Simulation. 1996. Prentice-Hall.

[41] Mehta, A.. Smart Modeling: Basic Methodology and Advanced Tools. Joines, J. A. Barton R. R. Kang K. Fishwick A. Proceedings ofthe winter simulation conference , 241245. 2000.

\footnotetext{
${ }^{i}$ http://www.straumann.nl/news-nl-07032011-itero-prijslijst-nl-2011.pdf
} 